\title{
Research and Development Strategies for Hybrid japonica Rice
}

Wenjing Zheng ${ }^{1+} \mathbb{0}$, Zuobin $\mathrm{Ma}^{1+}$, Mingzhu Zhao ${ }^{1}$, Minggang Xiao², Jiaming Zhao ${ }^{3}$, Changhua Wang ${ }^{1}$, Hong Gao', Yuanjun Bai', Hui Wang ${ }^{3}$ and Guomin Sui ${ }^{3^{*}}$

\begin{abstract}
The utilization of heterosis has resulted in significant breakthroughs in rice breeding. However, the development of hybrid japonica has been slow in comparison with that of hybrid indica. The present review explores the history and current status of hybrid japonica breeding. With the creation of japonica cytoplasmic male sterility and photothermo-sensitive genic male sterile lines, both three-line and two-line systems of hybrid rice have been created, and a series of hybrid japonica rice varieties have been developed and cultivated widely. At the same time, some progress has been made in genetic research of molecular mechanism for heterosis and QTL mapping for traits such as fertility, stigma exposure and flower time. In addition, genomics and transcriptome have been widely used in the research of hybrid rice, which provides a strong support for its development. Although the research on hybrid japonica has made many advances, there are still some restrictive problems. Based on the research and production of hybrid japonica rice, the prospect and development strategies of hybrid japonica rice are analyzed.
\end{abstract}

Keywords: Hybrid Japonica Rice, Three-line, Two-line, Heterosis

\section{Introduction}

Among more than 120 rice-producing countries, over $95 \%$ of them cultivate primarily indica varieties (Deng 2008; Fang 2005). Globally, only a few countries, such as China, Japan, South Korea, United States, Australia, and Egypt, produce and export japonica rice (Deng 2008). The japonica varieties occupy $8.8 \%$ of the rice farming area and are responsible for $14.2 \%$ of total rice production worldwide (Fang 2005). From 2007 to 2015, the total amount of rice traded on international markets increased from 32 million tons to 41.67 million tons, while the proportion of japonica rice trade decreased during the same time from $10.9 \%$ to $8.1 \%$. This decrease corresponds to approximately 3.5 million tons, which is less than $5 \%$ of the amount of japonica rice consumed in China (Cao et al. 2018). China has the largest planting area and the highest total production of japonica rice in

\footnotetext{
* Correspondence: guomin666@126.com

tWenjing Zheng and Zuobin Ma contributed equally to this work

${ }^{3}$ Liaoning Academy of Agricultural Sciences, Shenyang 110161, China

Full list of author information is available at the end of the article
}

the world. Because the culinary quality of japonica is superior to that of the indica rice, the total production and demand for japonica rice has been increasing continuously in China, especial in Northeast China from 1990 to 2015 (Fig. 1, Fig. 2) (Tang et al. 2017). To meet the growing need, the share of the japonica in overall rice planting has been increasing recently, particularly in south China, the main indica producing area of the country.

The utilization of heterosis resulted in a major breakthrough in rice breeding, and the growing popularity of hybrid rice contributed significantly to world food production (Yuan 1987). In 1970, the wild abortive cytoplasm in rice was found in Hainan province, China, and in 1976, hybrid rice cultivars were released to farmers. In 1991, the area under hybrid rice was 17.6 million hectares, i.e., $55 \%$ of the total area occupied by hybrid rice in China. In particular, the current area of hybrid indica cultivation comprises more than $50 \%$ of the rice planting area (Pu et al. 2015). In contrast, the development of hybrid japonica was very slow, as it occupied 


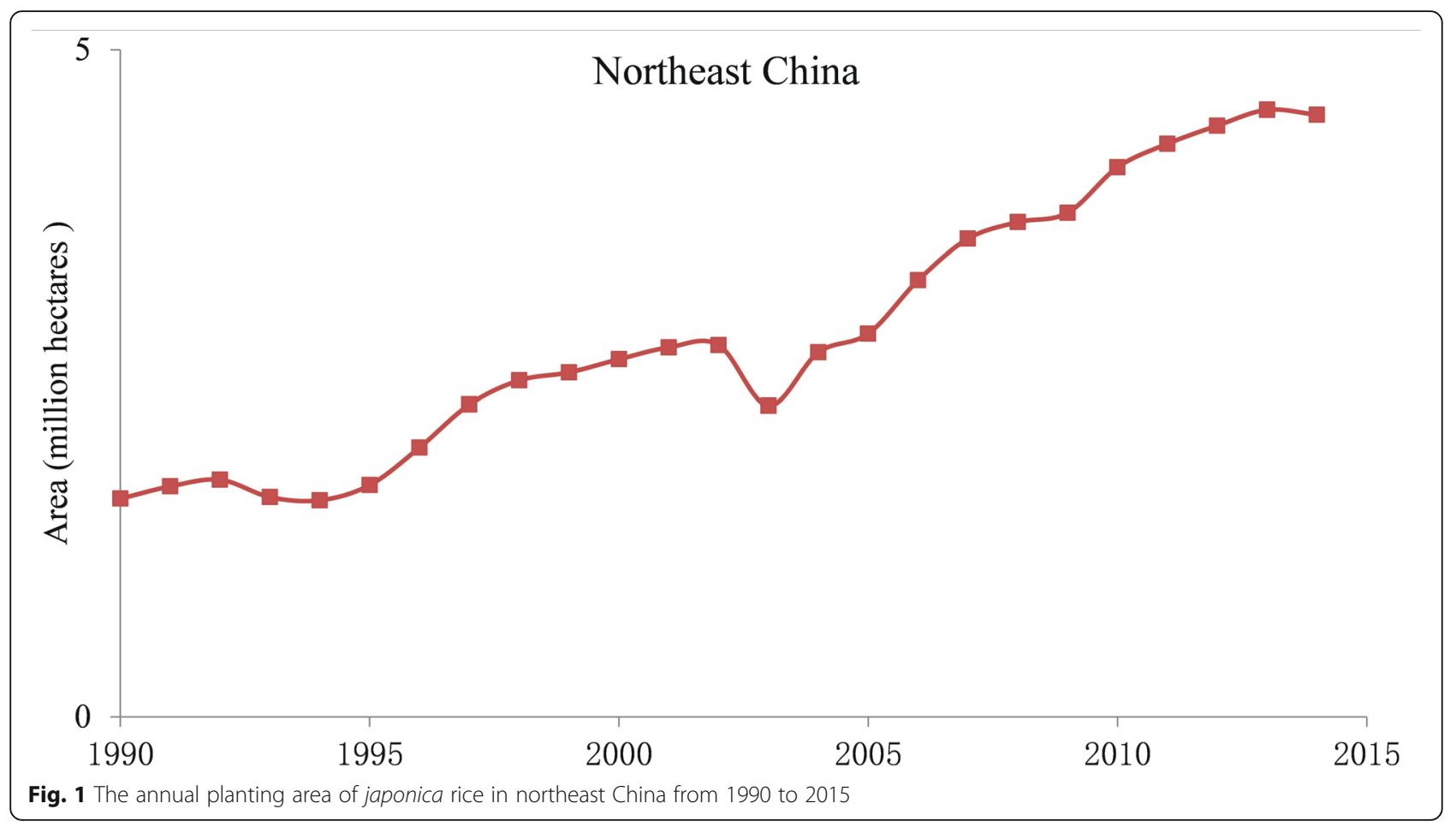

less than $3 \%$ of the total planting area of japonica (Deng 2008). As the development of hybrids offers a great potential to increase the yield of rice, Yuan (2000) predicted that hybrid japonica is the most likely to make a breakthrough in the next 30 years, which will lead to a new growth point in China's grain production. To further facilitate the development of the hybrid japonica rice, we have reviewed recent progress in research on the breeding of hybrid japonica and analyzed existing problems, thus providing a reference for the development of hybrid japonica rice.

\section{The History of Research on Hybrid japonica Rice}

The research on hybrid japonica rice was originated in Japan (Li and Wu 1991). In the 1950s, Weeraratne and Sampath first reported the phenomenon of cytoplasmic male sterility (CMS) in rice (Sampath and Mohauty, 1954). In 1958, CMS lines with the wild rice cytoplasm

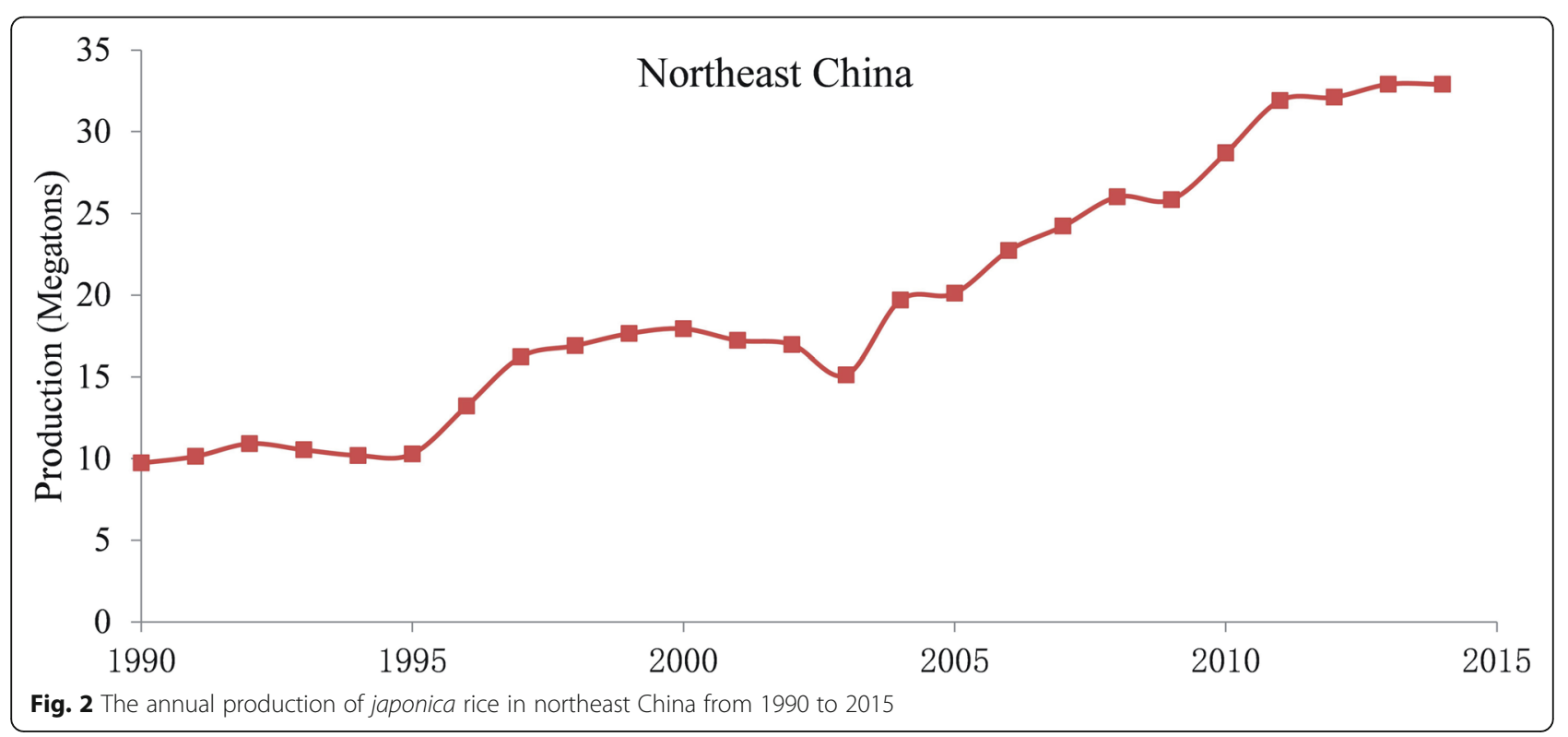


were obtained for the first time by Katsuo and Mizushima by backcrossing Fujisaka 5 and Chinese red-awned wild rice; however, no restorer lines were found at that time (Deng 2008). In 1966, Shinjyo and O'mura developed the first CMS lines of Baotai (BT) type in the backcross of Chinsurah Bolo II indica rice from India and Taichung 65 from Taiwan and found a few conspecific restorer lines, but due to the inconspicuous heterosis, the three-line varieties could not be widely promoted (Shiniyo C, O' mura 1966; Shiniyo C 1969, 1972). Subsequently, Watanabe developed the CMS-Lead lines in the cross of Lead (Myanmar indica) and Fujisaka 5 (Deng 2008). American and Indian researchers developed japonica CMS lines with cytoplasm from Taiwan cultivars Birco and Oryza glaberrima (Deng 2008). The International Rice Research Institute (IRRI) launched the hybrid rice research program in 1977, and after more than 10 years of effort, they developed the first batch of hybrid rice parents and hybrid rice varieties in 1989. With the help of international organizations and China, the technology and the material were transferred to some rice-producing countries in Asia to develop the production of hybrid rice (Huang 2004).

At present, there are more than 120 rice-cultivating countries on five continents. Outside of China, 110 million ha of rice are cultivated worldwide, mostly in Asia, Africa, and the Americas (Deng 2008; Hu 2010), and the area occupied by hybrid rice reached 6.36 million ha in 2014. Of these, 5.91 million ha were in Asia, and 0.45 million ha were in South and North America. The countries growing hybrid rice included Bangladesh, Pakistan, India, Indonesia, the Philippines, Myanmar, Vietnam, Sri Lanka, Iran, United States, Brazil, Argentina and Uruguay. China, India, Bangladesh, Pakistan, Indonesia, the Philippines, Myanmar, Vietnam, and the United States are the major countries growing hybrid rice. Hybrid rice in the United States accounts for more than half of its total rice production area. However, as the indica hybrid was the dominant rice in these countries, the breeding and promotion of hybrid japonica rice were limited (Xie and Peng 2016).

\section{Research Progress of Hybrid japonica Rice in China}

Since the 1960s, breeders led by Li Z and Yang Z have been carrying out research on hybrid japonica rice ( $\mathrm{Li}$ 1977, 2000; Li and Wu JL 1991; Yang 1994, 1998, 1999, $2005,2016)$. Over the past 50 years, remarkable progress has been made in the breeding of hybrid japonica rice. By 2019, China has approved 396 hybrid japonica rice combinations, whose parents, variety types, and time of being certified are listed in Table 1 . As can be seen from Table 1 and Fig. 3, most of the varieties were generated between 2000 and 2019, and the hybrid japonica rice accounts for $88.4 \%$ of the total number of cultivars, while indica-japonica varieties for only $11.6 \%$. Among these hybrid japonica rice varieties, the majority (360) are three-line varieties, and only 36 are two-line varieties.

\section{A Three-Line System for Hybrid Japonica Rice}

The three-line system is composed of sterile line, maintainer line, and restorer line. The discovery of sterile lines enabled large-scale production of hybrid rice. The key to the breeding of hybrid japonica rice is finding suitable restorer lines that can restore the male sterile lines and improve the heterosis.

\section{Dian-Type Male Sterile Lines}

The study of hybrid japonica rice in China began in 1965. A naturally sterile plant was found in the Taipei 8 , and was used as a female to develop the CMS-Dian I via nuclear replacement from japonica cultivar Hongmaoying in 1969. CMS-Dian I was the first and the most significant japonica CMS line in China (Li 2000; Yang 2016). Subsequently, japonica CMS lines, such as DianII, Dian-III, Dian-IV, Dian-V, Dian-VI, Dian-VII, and Dian-VIII were generated in the same manner. Although the lack of restorer lines limited the application of these CMS-Dian lines in the three-line system, they formed the foundation for the development of japonica hybrid rice in southwest, northwest, and eastern provinces of China (Li 1977). With the breakthrough achievement of creating Dian-type hybrid japonica rice, a number of stable CMS lines (Dianyu 1A, Dianxun 1A, Liyu A, and Yumi 15A) were developed, which were further used to create other hybrid japonica varieties, such as Yuza 29, Xunza 36, Dianza 32, Dianza 31, Yunguang 8, Yunguang 9, Yunguang 12, and Yunguang 14. These varieties not only exhibit a higher production of hybrid seeds but also display stronger heterosis and higher resistance to the blast disease in the $F_{1}$ generation (Huang 2004; Yang 2016).

\section{BT-Type Male Sterile Lines}

In 1972, the CMS-BT line Taichung 65 was introduced from Japan by the Liaoning Academy of Agricultural Sciences (LAAS) and the Chinese Academy of Agricultural Sciences. This line was used as the female parent to develop several CMS-BT lines, such as Liming A, Xiuling A, Akihikari A, Sasanishiki A, Liuqiangxin A, Sidao 8A, Wuyunjing 7A, Hanfeng A, Xiushui 4A, Zhong 7941A, 41A, Ning 67, and Yonging 2A. However, all these lines lacked appropriate japonica restorer lines (Deng 2008). In 1975, using the "indica-japonica bridging technique", LAAS introduced restorer genes from indica to japonica by backcross of IR8 / Keqing 3 // Keqing 3. As a result, the japonica restorer line C57 with 
Table 1 List of hybrid japonica rice varieties bred in China

\begin{tabular}{|c|c|c|c|c|c|}
\hline Vareties & Female & Male & Type & Subspecies & Year of release \\
\hline Li you 57 & Li ming $A$ & C57 & Three-lines & Japonica & 1980 \\
\hline Xiu you 57 & Xiu ling $A$ & C57 & Three-lines & Japonica & 1984 \\
\hline Dang you C bao & Dang xua nwan 3 A & C Bao & Three-lines & Japonica & 1985 \\
\hline Di you 57 & D $57 \mathrm{~A}$ & C57-10 & Three-lines & Japonica & 1985 \\
\hline Ji jing za 1 & Li ming $A$ & K55 & Three-lines & Japonica & 1986 \\
\hline Qiu you 20 & Qiu guang A & F20 & Three-lines & Japonica & 1986 \\
\hline Yan you 57 & Yan jing 903 A & C57V & Three-lines & Japonica & 1988 \\
\hline Han you xiang qing & Han feng A & xiang qing & Three-lines & Japonica & 1989 \\
\hline Liu you 3-2 & Liu qian xin A & ning hui 3-2 & Three-lines & Japonica & 1989 \\
\hline Dang you 9 & Dang xuan wan $2 \mathrm{~A}$ & wan hui 9 & Three-lines & Japonica & 1989 \\
\hline Feng you 9 & Feng jin A & C79-64 & Three-lines & Japonica & 1989 \\
\hline 76 you 312 & $76-27 \mathrm{~A}$ & pei C312 & Three-lines & Japonica & 1990 \\
\hline Han you 1027 & Han feng $A$ & T1027 & Three-lines & Japonica & 1990 \\
\hline Ai you 82 & Dong jiu ai $4 \mathrm{~A}$ & hong yu 82 & Three-lines & Japonica & 1990 \\
\hline Qi you 6 & $76-27 \mathrm{~A}$ & 2674 & Three-lines & Japonica & 1990 \\
\hline Xun za 29 & Dian xun 1 A & nan 29 & Three-lines & Japonica & 1991 \\
\hline Wan dao 16 & Liu qian xin A & Cbao & Three-lines & Japonica & 1992 \\
\hline Xin dao 4 & - & - & Three-lines & Japonica & 1992 \\
\hline Xin dao 3 & Liao 10120A & hui $73-28$ & Three-lines & Japonica & 1992 \\
\hline Wan dao 18 & Liu qian xin A & 82,022 & Three-lines & Japonica & 1992 \\
\hline Xu you 3-2 & Xu dao 2 A & ning hui 3-2 & Three-lines & Japonica & 1993 \\
\hline Si you 422 & Si dao $8 \mathrm{~A}$ & lun hui 422 & Three-lines & Japonica & 1993 \\
\hline Jing you 6 & Zhong zuo 59 A & jin 1244-2 & Three-lines & Japonica & 1993 \\
\hline Wan dao 26 & 70015 & xiu shui 04 & Two-lines & Japonica & 1994 \\
\hline Wan dao 22 & $80-4 \mathrm{~A}$ & wan hui 9 & Three-lines & Japonica & 1994 \\
\hline 70 you 9 & 70015 & wan hui 9 & Two-lines & Japonica & 1994 \\
\hline Ning you 1 & $552 \mathrm{~A}$ & FR-79 & Three-lines & Japonica & 1994 \\
\hline Si you 9083 & Si dao 8 A & C9083 & Three-lines & Japonica & 1994 \\
\hline E jing za 1 & N5088S & R187 & Two-lines & Japonica & 1995 \\
\hline 8 you 161 & $8204 \mathrm{~A}$ & R161 & Three-lines & Japonica & 1995 \\
\hline Hua jing za 1 & 70015 & R1514 & Two-lines & Japonica & 1995 \\
\hline Yu za 29 & Dian yu 1 A & nan 29-1 & Three-lines & Japonica & 1995 \\
\hline Wan dao 34 & $80-4 \mathrm{~A}$ & HP121 & Three-lines & Japonica & 1996 \\
\hline 9 you 138 & Xu 9201 A & N138 & Three-lines & Japonica & 1996 \\
\hline Liu you 3 & Liu gian xin A & yin hui 3 & Three-lines & Japonica & 1996 \\
\hline Qi you7 & $76-27$ A & K1457 & Three-lines & Japonica & 1996 \\
\hline Wan dao 48 & 70015 & shuang jiu & Two-lines & Japonica & 1997 \\
\hline Si you 9022 & Sidao $8 \mathrm{~A}$ & C9022 & Three-lines & Japonica & 1997 \\
\hline Qiu you 62 & Qiu guang A & C9162 & Three-lines & Japonica & 1997 \\
\hline Wan dao 46 & $80-4 \mathrm{~A}$ & T1027 & Three-lines & Japonica & 1997 \\
\hline Ti you 418 & Ti jin A & C418 & Three-lines & Japonica & 1998 \\
\hline Min you 128 & $83 \mathrm{~A}$ & R128 & Three-lines & Japonica & 1998 \\
\hline Liu you 121 & Liu gian xin A & HP121 & Three-lines & Japonica & 1998 \\
\hline Si you 88 & Sidao 8 A & hui 88 & Three-lines & Japonica & 1998 \\
\hline
\end{tabular}


Table 1 List of hybrid japonica rice varieties bred in China (Continued)

\begin{tabular}{|c|c|c|c|c|c|}
\hline Vareties & Female & Male & Type & Subspecies & Year of release \\
\hline Si you 418 & Sidao $8 \mathrm{~A}$ & C418 & Three-lines & Japonica & 1999 \\
\hline Wan dao 50 & 40085 & xiu shui 04 & Two-lines & Japonica & 1999 \\
\hline si you 523 & Sidao $8 \mathrm{~A}$ & R523 & Three-lines & Japonica & 1999 \\
\hline jin jing za 1 & LS2S & zhong zuo93 & Two-lines & Japonica & 1999 \\
\hline 9 you 418 & Xu 9201 A & C418 & Three-lines & Japonica & 2000 \\
\hline yong you 1 & Ning 67 A & K1722 & Three-lines & Japonica & 2000 \\
\hline ning you 2 & $401 \mathrm{~A}$ & R253 & Three-lines & Japonica & 2000 \\
\hline yun guang 8 & N5088S & yun hui 11 & Two-lines & Japonica & 2000 \\
\hline 8 you 682 & Xu 8908 A & R37682 & Three-lines & Japonica & 2000 \\
\hline 8 6you 8 & $863 \mathrm{~A}$ & ning hui 8 & Three-lines & Japonica & 2000 \\
\hline 3you 18 & $\operatorname{Jin} 3 A$ & C418 & Three-lines & Japonica & 2001 \\
\hline liao you 5218 & Liao 5216 A & C418 & Three-lines & Japonica & 2001 \\
\hline hua jing za 2 & N5088S & 41,678 & Two-lines & Japonica & 2001 \\
\hline yongyou 2 & Yongjing 2 A & K1722 & Three-lines & Japonica & 2001 \\
\hline siyou 12 & Sidao 8 A & Z12 & Three-lines & Japonica & 2001 \\
\hline liao you 3418 & Liao 326A & C418 & Three-lines & Japonica & 2001 \\
\hline jin jing za 3 & Zao hua dong A & c you 1 & Three-lines & Japonica & 2001 \\
\hline 69 you 8 & Xu 69A & R11238 & Three-lines & Japonica & 2001 \\
\hline yan you 1 & Yan jing 5 A & yan hui 93,005 & Three-lines & Japonica & 2001 \\
\hline liao you 4418 & Xiu ling A & C418 & Three-lines & Japonica & 2001 \\
\hline liao you 5 & Liao yan 28 A & $504-6$ & Three-lines & Japonica & 2001 \\
\hline jin jing za 2 & $\operatorname{Jin} 3 A$ & C272 & Three-lines & Japonica & 2001 \\
\hline IIlyou 98 & $\mathrm{MH} 2003 \mathrm{~A}$ & R18 & Three-lines & Japonica & 2002 \\
\hline chang you 1 & Wu yun jing $7 \mathrm{~A}$ & shen hui 254 & Three-lines & Japonica & 2002 \\
\hline yongyou 3 & Yongjing $2 \mathrm{~A}$ & K1863 & Three-lines & Japonica & 2002 \\
\hline dian za 31 & Yu mi $15 \mathrm{~A}$ & nan 34 & Three-lines & Japonica & 2002 \\
\hline shen you 1 & $8204 \mathrm{~A}$ & shen hui 1 & Three-lines & Japonica & 2002 \\
\hline liu you 8 & Liu gian xin A & HP121-8 & Three-lines & Japonica & 2002 \\
\hline dian za 32 & Li yu A & nan 34 & Three-lines & Japonica & 2002 \\
\hline minyou 55 & $261 S$ & $\min 55$ & Two-lines & Japonica & 2002 \\
\hline jin jing za 4 & $502 \mathrm{~A}$ & R411 & Three-lines & Japonica & 2002 \\
\hline liao you 1518 & Liao $151 \mathrm{~A}$ & C418 & Three-lines & Japonica & 2002 \\
\hline yun guang 9 & 70015 & yun hui 124 & Two-lines & Japonica & 2002 \\
\hline liao you 0201 & Liao 02 A & C01 & Three-lines & Japonica & 2002 \\
\hline pu you 801 & $69 \mathrm{~A}$ & $J 60$ & Three-lines & Japonica & 2002 \\
\hline 86you 242 & $863 \mathrm{~A}$ & R242 & Three-lines & Japonica & 2002 \\
\hline ba you 8 & $8204 \mathrm{~A}$ & R9525 & Three-lines & Japonica & 2002 \\
\hline xin za jing 1 & Pei ai 645 & yu jing 3 & Two-lines & Japonica & 2003 \\
\hline jin jing za 5 & Zao hua dong A & 773 & Three-lines & Japonica & 2003 \\
\hline xiang you 18 & Ai zhi xiang $A$ & MR18 & Three-lines & Japonica & 2003 \\
\hline liang you pei jing & Pei ai 645 & 94,205 & Two-lines & Japonica & 2003 \\
\hline ning you 3 & Zhong zuo59 A & 1229 & Three-lines & Japonica & 2003 \\
\hline wan dao 74 & $80-4 \mathrm{~A}$ & wan hui 98 & Three-lines & Japonica & 2003 \\
\hline yan you 2 & Yan 93,538 A & lun hui 422 & Three-lines & Japonica & 2003 \\
\hline
\end{tabular}


Table 1 List of hybrid japonica rice varieties bred in China (Continued)

\begin{tabular}{|c|c|c|c|c|c|}
\hline Vareties & Female & Male & Type & Subspecies & Year of release \\
\hline jing you 15 & Zhong zuo 59 A & Y772 & Three-lines & Japonica & 2003 \\
\hline shen you 4 & Shen $4 \mathrm{~A}$ & xiang qing & Three-lines & Japonica & 2003 \\
\hline liao you 14 & Liao $30 \mathrm{~A}$ & C4115 & Three-lines & Japonica & 2003 \\
\hline yun guang 12 & $95076 \mathrm{~S}$ & yun hui 124 & Two-lines & Japonica & 2003 \\
\hline minyou xiang jing & $261 S$ & Wxiang 99,075 & Two-lines & Japonica & 2003 \\
\hline yan liang you 2818 & GBO28S & C418 & Two-lines & Japonica & 2003 \\
\hline jin you 2003 & $341 \mathrm{~A}$ & 773 & Three-lines & Japonica & 2003 \\
\hline wan dao 72 & $80-4 \mathrm{~A}$ & 2277 & Three-lines & Japonica & 2003 \\
\hline yong you 4 & Yong jing $2 \mathrm{~A}$ & K2001 & Three-lines & Japonica & 2003 \\
\hline wan dao 70 & $80-4$ A & MR19 & Three-lines & Japonica & 2003 \\
\hline dong jing za 3 & N5088S & minhui 128 & Two-lines & Japonica & 2004 \\
\hline liao you 16 & Liao 30 A & C272 & Three-lines & Japonica & 2004 \\
\hline wan dao 80 & Shuang jiu A & wan hui 3402 & Three-lines & Japonica & 2004 \\
\hline wan dao 78 & YA & $9 \mathrm{M} 059$ & Three-lines & Japonica & 2004 \\
\hline yu za 34 & Dian yu $1 \mathrm{~A}$ & nan 34 & Three-lines & Japonica & 2004 \\
\hline wan han you 1 & N422S & R8272 & Two-lines & Japonica & 2004 \\
\hline dian za 33 & Yu mi $15 \mathrm{~A}$ & dian nong R-3 & Three-lines & Japonica & 2004 \\
\hline wan dao 76 & Ai zhi xiang A & MC20518 & Three-lines & Japonica & 2004 \\
\hline jing you 14 & Zhong zuo 59 A & jin dao 1229 & Three-lines & Japonica & 2004 \\
\hline shen you 254 & Shen $6 \mathrm{~A}$ & shen hui 254 & Three-lines & Japonica & 2004 \\
\hline 10 you 18 & $10 \mathrm{~A}$ & R148 & Three-lines & Japonica & 2004 \\
\hline yong you 6 & Yongjing 2 A & K4806 & Three-lines & Japonica & 2005 \\
\hline xiu you 5 & Xiu shui $110 \mathrm{~A}$ & xiu hui 69 & Three-lines & Japonica & 2005 \\
\hline jia you 1 & Jia 60 A & jia hui 40 & Three-lines & Japonica & 2005 \\
\hline liao you 1052 & $105 \mathrm{~A}$ & C52 & Three-lines & Japonica & 2005 \\
\hline jia le you 2 & $151 \mathrm{~A}$ & $\mathrm{DH} 32$ & Three-lines & Japonica & 2005 \\
\hline liao you 853 & Nong lin $150 \mathrm{~A}$ & R853 & Three-lines & Japonica & 2005 \\
\hline chang you 3 & Wu yun jing $7 \mathrm{~A}$ & R192 & Three-lines & Japonica & 2005 \\
\hline yong you 5 & Yong nuo $2 \mathrm{~A}$ & K6926 & Three-lines & Japonica & 2005 \\
\hline jing you 13 & Zhong zuo 59 A & lu hui 3 & Three-lines & Japonica & 2005 \\
\hline zhe you 9 & $5016 \mathrm{~A}$ & zhe hui 9816 & Three-lines & Japonica & 2005 \\
\hline shen you 693 & Shen $6 \mathrm{~A}$ & R693 & Three-lines & Japonica & 2005 \\
\hline xu you 201 & Xu 9320 A & xu hui 201 & Three-lines & Japonica & 2005 \\
\hline su you 22 & Wu yun jing $7 \mathrm{~A}$ & R16189 & Three-lines & Japonica & 2005 \\
\hline zhong jing you 1 & $\operatorname{Jin} 6 A$ & jin hui 1 & Three-lines & Japonica & 2005 \\
\hline chang you 2 & Wu yun jing 7 A & C53 & Three-lines & Japonica & 2005 \\
\hline liao you 2006 & Liao $20 \mathrm{~A}$ & C2106 & Three-lines & Japonica & 2005 \\
\hline yong you 8 & Yong jing $3 \mathrm{~A}$ & K6876 & Three-lines & Japonica & 2006 \\
\hline qiu you jin feng & Qiu feng A & R44 & Three-lines & Japonica & 2006 \\
\hline ai you 39 & Ai zhi xiang A & MR39 & Three-lines & Japonica & 2006 \\
\hline shen you 8 & Shen $4 \mathrm{~A}$ & R8 & Three-lines & Japonica & 2006 \\
\hline dian za 80 & Dian I-11A & nan 34 & Three-lines & Japonica & 2006 \\
\hline ling xiang you 18 & Ling xiang A & YC418 & Three-lines & Japonica & 2006 \\
\hline jin jing you 68 & $\operatorname{Jin} 1007 \mathrm{~A}$ & jin hui 68 & Three-lines & Japonica & 2006 \\
\hline
\end{tabular}


Table 1 List of hybrid japonica rice varieties bred in China (Continued)

\begin{tabular}{|c|c|c|c|c|c|}
\hline Vareties & Female & Male & Type & Subspecies & Year of release \\
\hline yongyou 1460 & Yong jing $2 \mathrm{~A}$ & T1460 & Three-lines & Japonica & 2006 \\
\hline wan dao 88 & $9201 \mathrm{~A}$ & R-8 & Three-lines & Japonica & 2006 \\
\hline jin jing you 88 & Jin $1007 \mathrm{~A}$ & jin hui 88 & Three-lines & Japonica & 2006 \\
\hline ba you 52 & $8204 \mathrm{~A}$ & Z052 & Three-lines & Japonica & 2006 \\
\hline liao you 2016 & Liao 20 A & C216 & Three-lines & Japonica & 2006 \\
\hline liao you 2015 & Liao 20 A & C4115 & Three-lines & Japonica & 2006 \\
\hline dian za 36 & He xi 42-7 A & nan 36 & Three-lines & Japonica & 2006 \\
\hline jin jing you 28 & $\operatorname{Jin} 1007 \mathrm{~A}$ & jin hui 28 & Three-lines & Japonica & 2006 \\
\hline liao you 5224 & Liao 5216 A & C124 & Three-lines & Japonica & 2006 \\
\hline dian za 35 & He xi 42-5 A & nan 34 & Three-lines & Japonica & 2006 \\
\hline ling feng you 18 & Ling feng A & SJR218 & Three-lines & Japonica & 2006 \\
\hline xu you 631 & Xu 9320A & xu hui 11,631 & Three-lines & Japonica & 2006 \\
\hline chun you 2 & Chun jiang $12 \mathrm{~A}$ & $\mathrm{CH} 89$ & Three-lines & Japonica & 2006 \\
\hline su jing you 2 & $8006 \mathrm{~A}$ & xiang qing & Three-lines & Japonica & 2006 \\
\hline jin you 2006 & Jin dao $341 \mathrm{~A}$ & C4115 & Three-lines & Japonica & 2006 \\
\hline yong you 9 & Yong jing 2 A & K6093 & Three-lines & Japonica & 2007 \\
\hline yong you 10 & Yong nuo $2 \mathrm{~A}$ & K6962 & Three-lines & Japonica & 2007 \\
\hline jia you 2 & Jia 60 A & jia hui 30 & Three-lines & Japonica & 2007 \\
\hline xu you 733 & Xu 364 A & xu hui 11,733 & Three-lines & Japonica & 2007 \\
\hline dian you 34 & Dian jing you 1 A & nan 34 & Three-lines & Japonica & 2007 \\
\hline chun you 58 & Chun jiang $12 \mathrm{~A}$ & $\mathrm{CH} 58$ & Three-lines & Japonica & 2007 \\
\hline jing za you 1 & $80-4 \mathrm{~A}$ & jing hui 1 & Three-lines & Japonica & 2007 \\
\hline chang you 4 & Wu yun jing $7 \mathrm{~A}$ & CR-25 & Three-lines & Japonica & 2007 \\
\hline shuang you 3404 & Shuang jiu A & wan hui 3404 & Three-lines & Japonica & 2007 \\
\hline tian xie 13 & Xu 9320 A & xu hui 11,733 & Three-lines & Japonica & 2007 \\
\hline shen you 9723 & Shen $97 \mathrm{~A}$ & R4023 & Three-lines & Japonica & 2007 \\
\hline jin jing you 180 & $\operatorname{Jin} 5 A$ & R180 & Three-lines & Japonica & 2007 \\
\hline su jing you 3 & $9703 \mathrm{~A}$ & xiang qing & Three-lines & Japonica & 2007 \\
\hline yong you 11 & Yong jing 2 A & K216211 & Three-lines & Japonica & 2007 \\
\hline liao you 5273 & Liao 5216 A & $\mathrm{C} 73$ & Three-lines & Japonica & 2007 \\
\hline zhong jing you 470 & Zhong zuo $59 \mathrm{~A}$ & C470 & Three-lines & Japonica & 2007 \\
\hline xiu you 169 & jia hua 1 A & XR69 & Three-lines & Japonica & 2007 \\
\hline Tyou 5 & $951 \mathrm{~A}$ & R981 & Three-lines & Japonica & 2007 \\
\hline 5 you 135 & jin $5 \mathrm{~A}$ & C135 & Three-lines & Japonica & 2007 \\
\hline jin jing you 116 & jin $6 \mathrm{~A}$ & R116 & Three-lines & Japonica & 2007 \\
\hline hua you 14 & shen $9 \mathrm{~A}$ & fan 14 & Three-lines & Japonica & 2008 \\
\hline xin dao 22 & $\mathrm{LA} 3$ & LC64 & Three-lines & Japonica & 2008 \\
\hline ti you 267 & ti jin $A$ & C267 & Three-lines & Japonica & 2008 \\
\hline 5 you 280 & jin $5 \mathrm{~A}$ & R280 & Three-lines & Japonica & 2008 \\
\hline qiu you 118 & qiu feng $A$ & R118 & Three-lines & Japonica & 2008 \\
\hline 5 you 190 & jin $5 \mathrm{~A}$ & R190 & Three-lines & Japonica & 2008 \\
\hline zhong jing you 8 & jin jing $12 \mathrm{~A}$ & jin hui 3 & Three-lines & Japonica & 2008 \\
\hline fu you 135 & fu A & C135 & Three-lines & Japonica & 2008 \\
\hline xu 2you 1 & $x u 20,111 \mathrm{~A}$ & xu hui 201 & Three-lines & Japonica & 2008 \\
\hline
\end{tabular}


Table 1 List of hybrid japonica rice varieties bred in China (Continued)

\begin{tabular}{|c|c|c|c|c|c|}
\hline Vareties & Female & Male & Type & Subspecies & Year of release \\
\hline 6you 160 & jin $6 \mathrm{~A}$ & R160 & Three-lines & Japonica & 2008 \\
\hline zhe jing you 1 & zhe jing $2 \mathrm{~A}$ & zhe jing hui 04-02 & Three-lines & Japonica & 2008 \\
\hline zhe you 10 & $8204 \mathrm{~A}$ & zhe hui 9816 & Three-lines & Japonica & 2008 \\
\hline xin 8 you 122 & xin $8 \mathrm{~A}$ & GR03122 & Three-lines & Japonica & 2008 \\
\hline zhe you 12 & zhe $04 \mathrm{~A}$ & zhe hui H414 & Three-lines & Japonica & 2008 \\
\hline chun you 59 & chun jiang 16 A & $\mathrm{CH} 59$ & Three-lines & Japonica & 2009 \\
\hline yun liang you 144 & 23015 & yun R144 & Two-lines & Japonica & 2009 \\
\hline jin 7you 18 & jin feng $7 \mathrm{~A}$ & jin hui 18 & Three-lines & Japonica & 2009 \\
\hline xiu you 378 & xiu shui $3 \mathrm{~A}$ & XR78 & Three-lines & Japonica & 2009 \\
\hline zhe jing you 2 & zhe jing $3 \mathrm{~A}$ & zhe jing hui 04-02 & Three-lines & Japonica & 2009 \\
\hline shen you fan 15 & shen $10 \mathrm{~A}$ & shen fan 15 & Three-lines & Japonica & 2009 \\
\hline chun you 172 & chun jiang $12 \mathrm{~A}$ & C172 & Three-lines & Japonica & 2009 \\
\hline liao you 9573 & liao $95 \mathrm{~A}$ & $\mathrm{C} 73$ & Three-lines & Japonica & 2009 \\
\hline jin 7 you 58 & jin feng $7 \mathrm{~A}$ & jin hui 58 & Three-lines & Japonica & 2009 \\
\hline 95 you 161 & $95,122 \mathrm{~A}$ & R161 & Three-lines & Japonica & 2009 \\
\hline chun you 658 & chun jiang 16 A & $\mathrm{CH} 58$ & Three-lines & Japonica & 2009 \\
\hline dian za 86 & D5 A & nan 34 & Three-lines & Japonica & 2009 \\
\hline xu you 502 & xu 8908 A & xu hui 502 & Three-lines & Japonica & 2009 \\
\hline zhong jing you 13 & jin jing 13 A & jin hui 3 & Three-lines & Japonica & 2009 \\
\hline yong you 14 & yong jing $3 \mathrm{~A}$ & F5006 & Three-lines & Japonica & 2009 \\
\hline 5 you 360 & jin $5 \mathrm{~A}$ & R360 & Three-lines & Japonica & 2009 \\
\hline dian za 40 & chu jing $23 \mathrm{~A}$ & nan 34 & Three-lines & Japonica & 2009 \\
\hline jia you 608 & jia $60 \mathrm{~A}$ & jia hui 82 & Three-lines & Japonica & 2009 \\
\hline 3 you 88 & jin $3 A$ & LC50-88 & Three-lines & Japonica & 2009 \\
\hline xin han you 26 & pei ai 645 & 99,026 & Two-lines & Japonica & 2009 \\
\hline liao you 1498 & $14 \mathrm{~A}$ & C198 & Three-lines & Japonica & 2009 \\
\hline yun you 948 & G2480 A & yun R948 & Three-lines & Japonica & 2009 \\
\hline dian za 501 & D5 A & $Y-11$ & Three-lines & Japonica & 2009 \\
\hline ji liao za you 1 & liao 99 A & C746 & Three-lines & Japonica & 2009 \\
\hline xin dao 25 & LA3 & LC109 & Three-lines & Japonica & 2009 \\
\hline jia you 3 & jia 335 A & jia hui 32 & Three-lines & Japonica & 2009 \\
\hline xu 68 you 201 & xu 91,068 A & xu hui 201 & Three-lines & Japonica & 2009 \\
\hline jin 9you 78 & jin feng $9 \mathrm{~A}$ & jin hui 78 & Three-lines & Japonica & 2009 \\
\hline dian za 41 & he $x i$ 42-7 A & nan 43 & Three-lines & Japonica & 2009 \\
\hline ba you 315 & $8204 \mathrm{~A}$ & zhe hui H315 & Three-lines & Japonica & 2009 \\
\hline zhong zhong you 2005 & $25 \mathrm{~A}$ & R18 & Three-lines & Japonica & 2009 \\
\hline yong you 12 & yong jing $2 \mathrm{~A}$ & F5032 & Three-lines & Japonica & 2010 \\
\hline yun guang 101 & N95076S & yun jing hui 1 & Two-lines & Japonica & 2010 \\
\hline jia le you 100 & $151 \mathrm{~A}$ & GR100 & Three-lines & Japonica & 2010 \\
\hline su you 72 & su $77 \mathrm{~A}$ & su hui 162 & Three-lines & Japonica & 2010 \\
\hline jin jing you 132 & jin jing $13 \mathrm{~A}$ & jin hui 2 & Three-lines & Japonica & 2010 \\
\hline chang you 5 & chang 01-11 A & CR-27 & Three-lines & Japonica & 2010 \\
\hline jing liang you 5975 & 02595 & gr75 & Two-lines & Japonica & 2010 \\
\hline han you 8 & hu han $2 \mathrm{~A}$ & xiang qing & Three-lines & Japonica & 2010 \\
\hline
\end{tabular}


Table 1 List of hybrid japonica rice varieties bred in China (Continued)

\begin{tabular}{|c|c|c|c|c|c|}
\hline Vareties & Female & Male & Type & Subspecies & Year of release \\
\hline zhong jing you 15 & jin jing $12 \mathrm{~A}$ & jin hui 5 & Three-lines & Japonica & 2010 \\
\hline yong you 13 & yong jing $3 \mathrm{~A}$ & F5032 & Three-lines & Japonica & 2010 \\
\hline dian za 37 & he $x i$ 42-7 A & yin hui 1 & Three-lines & Japonica & 2010 \\
\hline liao you 9906 & liao $99 \mathrm{~A}$ & C2106 & Three-lines & Japonica & 2010 \\
\hline yong you 7 & yong jing $3 \mathrm{~A}$ & K6262 & Three-lines & Japonica & 2010 \\
\hline long you 1715 & long 17 A & R1415 & Three-lines & Japonica & 2010 \\
\hline jia pu you 608 & jia $335 \mathrm{~A}$ & jia hui 52 & Three-lines & Japonica & 2010 \\
\hline bi jing za 2035 & BJ-1 A & ZC2035 & Three-lines & Japonica & 2010 \\
\hline shen you 1 & $5 \mathrm{~A}$ & C3 & Three-lines & Japonica & 2010 \\
\hline jing liang you 2847 & NC228S & R4769 & Two-lines & Japonica & 2010 \\
\hline jia you 5 & jia 335 A & jia hui 125 & Three-lines & Japonica & 2010 \\
\hline dian you 35 & $\mathrm{DHC}-10 \mathrm{~A}$ & nan 34 & Three-lines & Japonica & 2010 \\
\hline dan jing you 8 & dan jing $4 \mathrm{~A}$ & dan hui 8 & Three-lines & Japonica & 2011 \\
\hline dian za 46 & he xi 42-7 A & nan 46 & Three-lines & Japonica & 2011 \\
\hline jing you 558 & jing 139 A & R558 & Three-lines & Japonica & 2011 \\
\hline shen you 16 & shen $46 \mathrm{~A}$ & shen fan 16 & Three-lines & Japonica & 2011 \\
\hline yun guang 109 & N95076S & yun jing hui 7 & Two-lines & Japonica & 2011 \\
\hline yun guang 104 & N95076S & yun jing hui 4 & Two-lines & Japonica & 2011 \\
\hline xin jing you 1 & xin dao $97,200 \mathrm{~A}$ & xin hui 3 & Three-lines & Japonica & 2011 \\
\hline xin dao 38 & LA28 & LC109 & Three-lines & Japonica & 2011 \\
\hline yun guang 107 & yun jing $202 \mathrm{~s}$ & yun jing hui 7 & Two-lines & Japonica & 2011 \\
\hline jiao za jing 1 & jiao $31 \mathrm{~A}$ & jiao hui 2 & Three-lines & Japonica & 2011 \\
\hline dong jing you 775 & dong wan $17 \mathrm{~A}$ & jing xiang 75 & Three-lines & Japonica & 2011 \\
\hline dian za 94 & D5A & $Y-16$ & Three-lines & Japonica & 2011 \\
\hline yong you 17 & yong jing $4 \mathrm{~A}$ & yonghui 12 & Three-lines & Japonica & 2012 \\
\hline zhe you 18 & zhe $04 \mathrm{~A}$ & zhe hui 818 & Three-lines & Japonica-indica & 2012 \\
\hline dian you 38 & $\mathrm{DHC}-10 \mathrm{~A}$ & dian nong R-5 & Three-lines & Japonica & 2012 \\
\hline chun you 618 & chun jiang 16 A & $\mathrm{C} 18$ & Three-lines & Japonica & 2012 \\
\hline shuang you 18 & shuang jiu A & C418 & Three-lines & Japonica & 2012 \\
\hline shen you C9 & shen wu $1 \mathrm{~A}$ & shen hui C9 & Three-lines & Japonica & 2012 \\
\hline 6you 53 & $1586 S$ & xin jing 5003 & Two-lines & Japonica & 2012 \\
\hline bao jing za 2 & N95076S & BR-4 & Two-lines & Japonica & 2012 \\
\hline xin dao 40 & LRA3 & LRC64 & Three-lines & Japonica & 2012 \\
\hline dian za 701 & D5 A & dian kun xiang 4 & Three-lines & Japonica & 2012 \\
\hline jia he you 555 & jia he $212 \mathrm{~A}$ & jia he hui 555 & Three-lines & Japonica & 2012 \\
\hline jin hui you 50 & jin hui $A$ & JP50 & Three-lines & Japonica & 2012 \\
\hline qin na 1 & yan nong $S$ & hun he fu ben & Two-lines & Japonica & 2012 \\
\hline dong jing you 763 & dong wan $17 \mathrm{~A}$ & xiang hui 63 & Three-lines & Japonica & 2012 \\
\hline dian za 49 & he xi 42-7 A & nan 50 & Three-lines & Japonica & 2012 \\
\hline yongyou 16 & yong jing $8 \mathrm{~A}$ & yonghui 12 & Three-lines & Japonica & 2012 \\
\hline jia you 6 & jia 335 A & jia hui 69 & Three-lines & Japonica & 2012 \\
\hline dian you 37 & DHC-10 A & dian nong R-3 & Three-lines & Japonica & 2012 \\
\hline jia chang you 7 & qiao feng $A$ & hui 135 & Three-lines & Japonica & 2012 \\
\hline yong you 538 & yong jing $3 \mathrm{~A}$ & F7538 & Three-lines & Japonica-indica & 2013 \\
\hline
\end{tabular}


Table 1 List of hybrid japonica rice varieties bred in China (Continued)

\begin{tabular}{|c|c|c|c|c|c|}
\hline Vareties & Female & Male & Type & Subspecies & Year of release \\
\hline chun you 84 & chun jiang $16 \mathrm{~A}$ & C84 & Three-lines & Japonica & 2013 \\
\hline yongyou 2640 & yong jing 26 A & F7540 & Three-lines & Japonica-indica & 2013 \\
\hline gang you 1 & $071 \mathrm{~A}$ & C419 & Three-lines & Japonica & 2013 \\
\hline long jing 1550 & yan feng 475 & liao xing 1 & Two-lines & Japonica & 2013 \\
\hline wu you 17 & wu A & $\mathrm{C} 17$ & Three-lines & Japonica & 2013 \\
\hline yong you 1640 & yong jing $16 \mathrm{~A}$ & F7540 & Three-lines & Japonica & 2013 \\
\hline dian he you 34 & $\mathrm{H} 479 \mathrm{~A}$ & nan 34 & Three-lines & Japonica & 2013 \\
\hline yong you 720 & yong jing $7 \mathrm{~A}$ & yong hui 20 & Three-lines & Japonica & 2013 \\
\hline chang you jing 6 & chang $119 \mathrm{~A}$ & CR-312 & Three-lines & Japonica & 2013 \\
\hline zhe nuo you 1 & zhe nuo $1 \mathrm{~A}$ & zhe nuo hui 04-01 & Three-lines & Japonica & 2013 \\
\hline T12you 66 & T4012 A & R7066 & Three-lines & Japonica & 2013 \\
\hline jing liang you 5519 & N55s & R19 & Two-lines & Japonica & 2013 \\
\hline 18 you 75 & $18 \mathrm{~A}$ & R1575 & Three-lines & Japonica & 2013 \\
\hline pu you 22 & ai jing $15 \mathrm{~S}$ & pu hui 22 & Two-lines & Japonica & 2013 \\
\hline jin jing you 11 & jin jing $11 \mathrm{~A}$ & jin hui 1 & Three-lines & Japonica & 2013 \\
\hline ji you 1769 & T176 A & C269 & Three-lines & Japonica & 2013 \\
\hline tong you jing 1 & yang fu jing $7 \mathrm{~A}$ & R98 & Three-lines & Japonica & 2013 \\
\hline yongyou 1540 & yong jing $15 \mathrm{~A}$ & F7540 & Three-lines & Japonica-indica & 2014 \\
\hline zhe you 13 & zhe $04 \mathrm{~A}$ & zhe hui H813 & Three-lines & Japonica & 2014 \\
\hline ji you 3985 & $639 \mathrm{~A}$ & ji jing 85 & Three-lines & Japonica & 2014 \\
\hline yong you 1109 & yong jing $11 \mathrm{~A}$ & F7509 & Three-lines & Japonica & 2014 \\
\hline shen you 17 & shen wu $1 \mathrm{~A}$ & shen fan 17 & Three-lines & Japonica & 2014 \\
\hline IIlyou 304 & $2003 \mathrm{~A}$ & $\mathrm{XHO4}$ & Three-lines & Japonica & 2014 \\
\hline long you 467 & long $3 \mathrm{~A}$ & R467 & Three-lines & Japonica & 2014 \\
\hline dan jing you 1 & dan jing 4 A & dan hui 1 & Three-lines & Japonica & 2014 \\
\hline jing you 106 & jing 139 A & C2106 & Three-lines & Japonica & 2014 \\
\hline re jing you 35 & re jing $1 \mathrm{~A}$ & jing hui 35 & Three-lines & Japonica & 2014 \\
\hline jiao yuan you 69 & jiao yuan 5A & JP69 & Three-lines & Japonica & 2014 \\
\hline dian kun you 8 & K5 A & S8 & Three-lines & Japonica & 2014 \\
\hline yong you 362 & yong jing $5 \mathrm{~A}$ & F7562 & Three-lines & Japonica & 2014 \\
\hline chun you 149 & chun jiang 19 A & $\mathrm{CH} 149$ & Three-lines & Japonica & 2014 \\
\hline liao 16you 06 & liao 5216 A & C2106 & Three-lines & Japonica & 2014 \\
\hline liao 73you 62 & liao 73 A & C62 & Three-lines & Japonica & 2014 \\
\hline yong you 1538 & yong jing $15 \mathrm{~A}$ & F7538 & Three-lines & Japonica-indica & 2015 \\
\hline shen you 24 & shen $01 \mathrm{~A}$ & shen fan 24 & Three-lines & Japonica & 2015 \\
\hline dian he you 56 & yu mi $15 \mathrm{~A}$ & nan 56 & Three-lines & Japonica & 2015 \\
\hline 5 you 68 & $5 \mathrm{~A}$ & R68 & Three-lines & Japonica & 2015 \\
\hline dong nuo you 91 & dong nuo $19 \mathrm{~A}$ & nuo hui 11 & Three-lines & Japonica & 2015 \\
\hline yong you 4949 & yong jing $49 \mathrm{~A}$ & F9249 & Three-lines & Japonica-indica & 2015 \\
\hline 76 liang you 5 & $95076 \mathrm{~S}$ & bao hui 5 & Two-lines & Japonica & 2015 \\
\hline qiu you 336 & qiu $15 \mathrm{~A}$ & R336 & Three-lines & Japonica & 2015 \\
\hline dian he you 4106 & he $x i$ 42-7 A & yin hui 106 & Three-lines & Japonica & 2015 \\
\hline yong you 1140 & yong jing $6 \mathrm{~A}$ & F7540 & Three-lines & Japonica-indica & 2015 \\
\hline tian long you 619 & L6 A & R19 & Three-lines & Japonica & 2015 \\
\hline
\end{tabular}


Table 1 List of hybrid japonica rice varieties bred in China (Continued)

\begin{tabular}{|c|c|c|c|c|c|}
\hline Vareties & Female & Male & Type & Subspecies & Year of release \\
\hline yong you 7850 & yong jing $78 \mathrm{~A}$ & F9250 & Three-lines & Japonica-indica & 2015 \\
\hline dian he you 55 & yu mi $15 \mathrm{~A}$ & nan 55 & Three-lines & Japonica & 2015 \\
\hline jing you 586 & jing 139 A & C586 & Three-lines & Japonica & 2015 \\
\hline yong you 4350 & yong jing $43 \mathrm{~A}$ & F9250 & Three-lines & Japonica-indica & 2015 \\
\hline bi jing you 210 & bi jing $2 \mathrm{~A}$ & NR210 & Three-lines & Japonica & 2015 \\
\hline yong you 4550 & yong jing 45 A & F9250 & Three-lines & Japonica-indica & 2015 \\
\hline yong you 150 & yong jing $2 \mathrm{~A}$ & F9250 & Three-lines & Japonica-indica & 2016 \\
\hline bi jing you 3 & bi jing $2 \mathrm{~A}$ & bi jing hui 3 & Three-lines & Japonica & 2016 \\
\hline yong you 4901 & A49 & F8001 & Three-lines & Japonica-indica & 2016 \\
\hline jia you zhong ke 3 & jia $66 \mathrm{~A}$ & zhong ke jia hui 1293 & Three-lines & Japonica-indica & 2016 \\
\hline zhe you 21 & zhe $04 \mathrm{~A}$ & zhe hui F1121 & Three-lines & Japonica & 2016 \\
\hline dian he you 6612 & yu mi $15 \mathrm{~A}$ & nan 6612 & Three-lines & Japonica & 2016 \\
\hline jiao yuan you 1 & jiao yuan $3 \mathrm{~A}$ & jiao hui 1 & Three-lines & Japonica & 2016 \\
\hline yongyou 4149 & yong jing $41 \mathrm{~A}$ & F9249 & Three-lines & Japonica-indica & 2016 \\
\hline yongyou 4912 & yong jing $49 \mathrm{~A}$ & F7512 & Three-lines & Japonica-indica & 2016 \\
\hline pu you 201 & pu jing $06 \mathrm{~A}$ & T201 & Three-lines & Japonica-indica & 2016 \\
\hline jia he you 1 & jia he $212 \mathrm{~A}$ & hui SC01-1 & Three-lines & Japonica-indica & 2016 \\
\hline yong you 540 & yong jing $3 \mathrm{~A}$ & F7540 & Three-lines & Japonica-indica & 2016 \\
\hline yong you 7050 & A70 & F9250 & Three-lines & Japonica-indica & 2016 \\
\hline yong you 8050 & yong jing $80 \mathrm{~A}$ & F9250 & Three-lines & Japonica-indica & 2016 \\
\hline jia you zhong ke 1 & jia 66 A & zhong ke jia hui 1 & Three-lines & Japonica-indica & 2016 \\
\hline zi xiang you 24 & zi xiang A & shen fan 24 & Three-lines & Japonica & 2016 \\
\hline zhe you 19 & zhe $04 \mathrm{~A}$ & zhe hui F1015 & Three-lines & Japonica-indica & 2016 \\
\hline dian he you 6611 & yu mi $15 \mathrm{~A}$ & nan 6611 & Three-lines & Japonica & 2016 \\
\hline yong you 4953 & yong jing $49 \mathrm{~A}$ & F6853 & Three-lines & Japonica-indica & 2017 \\
\hline zhong jia you 6 & jia he 316 A & zhong hui 7206 & Three-lines & Japonica-indica & 2017 \\
\hline jin jing you 2018 & jin $20 \mathrm{~A}$ & jin hui 18 & Three-lines & Japonica & 2017 \\
\hline jia he you 7245 & jia he 212 A & zhong hui 7245 & Three-lines & Japonica & 2017 \\
\hline chun you 115 & chun jiang $16 \mathrm{~A}$ & $\mathrm{CH} 115$ & Three-lines & Japonica-indica & 2017 \\
\hline shen you 26 & shen $9 \mathrm{~A}$ & shen hui 26 & Three-lines & Japonica & 2017 \\
\hline qian jing you 57 & $163 \mathrm{~A}$ & NR210 & Three-lines & Japonica & 2017 \\
\hline jing you 165 & jing $139 \mathrm{~A}$ & C165 & Three-lines & Japonica & 2017 \\
\hline jia you zhong ke 6 & jia 66 A & zhong ke 6 & Three-lines & Japonica & 2017 \\
\hline qiu you 122 & qiu $9 \mathrm{~A}$ & R122 & Three-lines & Japonica & 2017 \\
\hline chang you 2 & chang jing $1 \mathrm{~A}$ & hui KF2 & Three-lines & Japonica-indica & 2017 \\
\hline chang you 312 & chang $132 \mathrm{~A}$ & CR-312 & Three-lines & Japonica & 2017 \\
\hline yong you 7860 & yong jing 78 A & F6860 & Three-lines & Japonica-indica & 2017 \\
\hline yong you 5552 & yong jing 55 A & F6852 & Three-lines & Japonica-indica & 2017 \\
\hline chun you 984 & chun jiang $99 \mathrm{~A}$ & C84 & Three-lines & Japonica-indica & 2017 \\
\hline tian long you 518 & long $5 \mathrm{~A}$ & C818 & Three-lines & Japonica & 2017 \\
\hline jin jing you 1918 & jin $19 \mathrm{~A}$ & jin hui 18 & Three-lines & Japonica & 2017 \\
\hline yong you 4543 & yong jing $45 \mathrm{~A}$ & F7543 & Three-lines & Japonica-indica & 2017 \\
\hline shen you 415 & shen $9 \mathrm{~A}$ & C415 & Three-lines & Japonica & 2017 \\
\hline lian 8 you 3 & lian $8 \mathrm{~A}$ & yun R3 & Three-lines & Japonica & 2017 \\
\hline
\end{tabular}


Table 1 List of hybrid japonica rice varieties bred in China (Continued)

\begin{tabular}{|c|c|c|c|c|c|}
\hline Vareties & Female & Male & Type & Subspecies & Year of release \\
\hline zhe jing you 1578 & zhe jing 7 A & zhe hui 1578 & Three-lines & Japonica & 2017 \\
\hline yong you 7861 & yong jing 78 A & F6861 & Three-lines & Japonica-indica & 2017 \\
\hline yong you 1662 & yong jing $16 \mathrm{~A}$ & F6862 & Three-lines & Japonica-indica & 2017 \\
\hline jing you 1 & jing 1 A & guang hui 1 & Three-lines & Japonica & 2017 \\
\hline hua zhong you 1 & hua zhong $1 \mathrm{~A}$ & hui 16 & Three-lines & Japonica-indica & 2017 \\
\hline jiao yuan you 5 & jiao yuan $3 \mathrm{~A}$ & jiao hui 5 & Three-lines & Japonica & 2017 \\
\hline yong you 5550 & yong jing $55 \mathrm{~A}$ & F9250 & Three-lines & Japonica-indica & 2017 \\
\hline 7you 1 & $7 \mathrm{~A}$ & yun R1 & Three-lines & Japonica & 2017 \\
\hline chun you 927 & chun jiang 16 A & C927 & Three-lines & Japonica-indica & 2017 \\
\hline jiao yuan you 6 & jiao yuan $2 \mathrm{~A}$ & jiao hui 6 & Three-lines & Japonica-indica & 2018 \\
\hline chun you 284 & chun jiang 23 A & C84 & Three-lines & Japonica & 2018 \\
\hline shen you 114 & shen $01 \mathrm{~A}$ & C14 & Three-lines & Japonica-indica & 2018 \\
\hline yong you 7753 & yong jing 77 A & F6853 & Three-lines & Japonica-indica & 2018 \\
\hline xiu you 7113 & xiu $71 \mathrm{~A}$ & XR13 & Three-lines & Japonica-indica & 2018 \\
\hline yong you 6760 & yong jing $67 \mathrm{~A}$ & F6860 & Three-lines & Japonica-indica & 2018 \\
\hline jiang liang you 7901 & jiang 795 & jiang hui 1501 & Two-lines & Japonica & 2018 \\
\hline qiu you 23 & qiu $23 \mathrm{~A}$ & R23 & Three-lines & Japonica & 2018 \\
\hline qian jing you 2 & $163 \mathrm{~A}$ & qian jing hui 2 & Three-lines & Japonica & 2018 \\
\hline shen wu you 26 & shen wu 1A & shen hui 26 & Three-lines & Japonica & 2018 \\
\hline shen 9you 09 & shen $9 \mathrm{~A}$ & shen hui 9 & Three-lines & Japonica & 2018 \\
\hline zhong he you 1 & jia he $212 \mathrm{~A}$ & NP001 & Three-lines & Japonica & 2018 \\
\hline jing you 653 & jing 65 A & C315 & Three-lines & Japonica & 2018 \\
\hline xiu you 207 & xiu shui 134 A & R207 & Three-lines & Japonica & 2018 \\
\hline zi xiang you 26 & zi xiang $A$ & shen hui 26 & Three-lines & Japonica & 2018 \\
\hline bi jing you 5 & $67 \mathrm{~A}$ & NR210 & Three-lines & Japonica & 2018 \\
\hline shu you 9 & jia 81 A & zhong ke jia hui 1308 & Three-lines & Japonica & 2019 \\
\hline pu jing you 701 & pu jing 06 A & PR701 & Three-lines & Japonica & 2019 \\
\hline chun you 584 & chun jiang 25 A & C84 & Three-lines & Japonica & 2019 \\
\hline chang you 998 & chang 386 A & CR998 & Three-lines & Japonica & 2019 \\
\hline yong you 6711 & yong jing 67 A & F5711 & Three-lines & Japonica-indica & 2019 \\
\hline jin liang you 852 & jin rui 85 & yun hui 503 & Two-lines & Japonica & 2019 \\
\hline yong you 1526 & yong jing $15 \mathrm{~A}$ & F4926 & Three-lines & Japonica-indica & 2019 \\
\hline shen you 27 & shen $10 \mathrm{~A}$ & shen hui 26 & Three-lines & Japonica & 2019 \\
\hline zhe jing you 6153 & zhe jing 7 A & zhe jing hui 6153 & Three-lines & Japonica & 2019 \\
\hline yong you 7053 & yong jing $70 \mathrm{~A}$ & F6853 & Three-lines & Japonica-indica & 2019 \\
\hline yong you 6763 & yong jing 67 A & F6863 & Three-lines & Japonica-indica & 2019 \\
\hline yun liang you 504 & yun jing 2085 & yun hui 504 & Two-lines & Japonica & 2019 \\
\hline pu jing you 201 & pu jing $06 \mathrm{~A}$ & PR201 & Three-lines & Japonica & 2019 \\
\hline liao 99you 30 & liao 99 A & C30 & Three-lines & Japonica & 2019 \\
\hline jia you 8 & jia 74 A & jia hui 8 & Three-lines & Japonica & 2019 \\
\hline yong you 5526 & yong jing $55 \mathrm{~A}$ & F4926 & Three-lines & Japonica-indica & 2019 \\
\hline chang you jing 7 & chang 410-2 A & CR-928 & Three-lines & Japonica & 2019 \\
\hline liao 99you 15 & liao 99 A & C415 & Three-lines & Japonica & 2019 \\
\hline yong you 5518 & yong jing 55 A & F4918 & Three-lines & Japonica-indica & 2019 \\
\hline
\end{tabular}




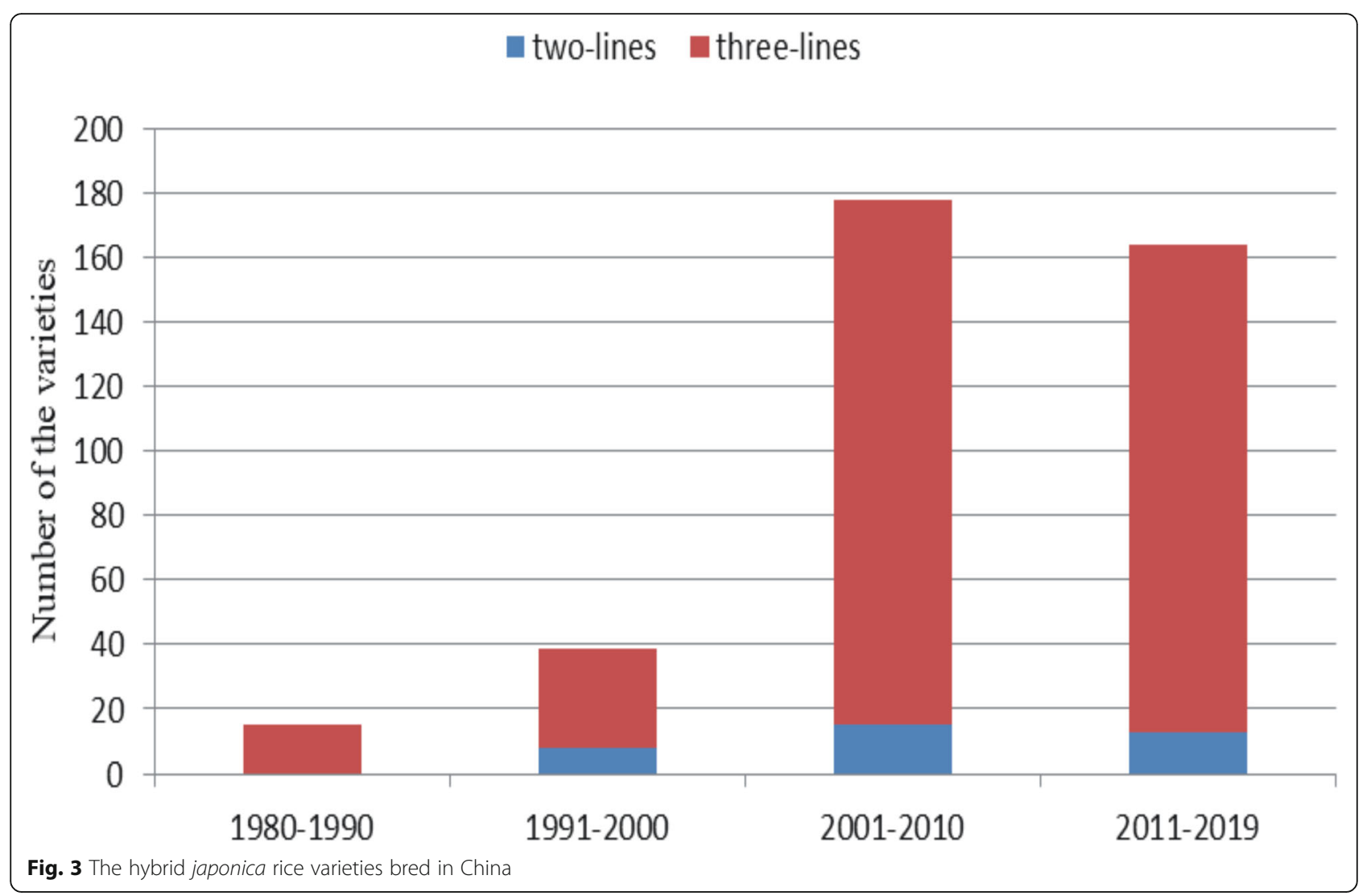

strong combining ability was developed, and the first hybrid japonica rice variety, Liyou 57, was successfully bred and was widely cultivated in China. This progress greatly promoted the research on hybrid japonica rice and its and utilization in northern China (Yang 1994). Many japonica restorer lines and their combinations were developed from C57 and its offspring for application in Beijing, Anhui, Zhejiang, Jiangsu, Hebei, Tianjin, and other provinces of China. This achievement resulted in a remarkable increase in the yield per unit area in northern China (Yang 2005). After the creation of the C57 line, the japonica restorer line C418 was developed by introducing wide-compatible genes, allowing to generate a series of hybrid rice combinations, such as Tiyou 418, Siyou 418, 9 You 418, and 3 You 18, and further promoting the development of hybrid rice (Yang 1998, 1999). The introduction of super-hybrid rice breeding project in 1998 drove continuous development in superhybrid japonica rice research (Hua et al. 2002, 2006). The seed production of japonica hybrid rice has been a difficult problem due to the delayed flowering time and low stigma exposure of japonica CMS lines. LAAS and National Japonica Research Center (NJRC) improved genetically the stigma exposure rate in sterile lines by introducing indica genes responsible for the high rate of stigma exertion. Using this strategy, a number of japonica CMS lines with high stigma exposure rates have been bred, which include Liao 105A, Liao 30A, Liao 02A, Liao 5216A, Liao 99A, Liao 60A, Liao 166A, Liao 11A, Liao 73A, and Liao 143A (Li 1987; Ling 1989; Shen et al. 1994; Wang et al. 2008). The National Japonica Engineering Technology Center (NJETC), China Rice Research Institute, and Jiangsu Academy of Agricultural Sciences have also developed japonica CMS lines with high stigma exposure rates, such as L6A, 18A, and Chunjiang 99A (Chen et al. 2011a; Dong et al. 2016). The genetic improvement of japonica CMS lines increased the stigma exposure rate from less than $30 \%$ to more than $60 \%$, reaching as high as $80 \%$ in some CMS lines (Wang et al. 2008). Further, the seed setting rate in natural outcrossings reached $40-60 \%$, and the seed production yield was markedly increased, from $1.5-2 \mathrm{t} /$ ha to 3.5-4.5 $\mathrm{t} / \mathrm{ha}$. Additionally, the flowering time of sterile lines started 30-60 min earlier, and the difference of flowering time between the parents shortened, so the problem of low seed production in hybrid japonica rice was solved (Wang et al. 2008). After the development of C418, a series of restorer lines with high quality, widecompatibility, and good combining ability were created by crossing early maturity japonica with ideotype in north China and tropical japonica in Southeast Asia. Examples of these lines are C2106, C787, C419, C315, C62, 
C224, C397, C4115, C52, C272, C73, C238, C415, R19, and LR27. These restorer parents with tropical japonica background are characterized not only by wide restoring spectrum but also strong restoring ability. These restorer parents exhibit several features of the ideal plant type, such as compact plant architecture, short flag leaf, and semidense or dense panicle type. In addition, quality, resistance, and maturity were also improved (Zhang et al. 1999). A series of high-quality super-hybrid japonica varieties developed using the newly developed CMS and restorer lines were released. For instance, the hybrid japonica varieties include the Liaoyou 5218, Liaoyou 1518, Liaoyou 16, Liaoyou 14, Liaoyou 5273, Liaoyou 0201, Liaoyou 1052, Liaoyou 9573, Liaoyou 1498, Liaoyou 20, Liaoyou 2006, Liaoyou 2015, Liaoyou 2106, Liaoyou 5206, Liaoyou 9906, Liao99you15, Liao99you27, Liao99you30, Liao16you06, Liao73you62, Jingyou558, Jingyou106, Jingyou165 and Jingyou653 released by LAAS (Wang 2008), Jinjingza 2 and Jinjingza 4 released by the Tianjin Academy of Agricultural Science, Gangyou 1 released by the Donggang Farm in Liaoning province, and the high-quality fragrant hybrid japonica rice Tianlongyou 619 released by the National japonica rice Engineering Technology Center (NJETC). In addition, a series of hybrid japonica rice, such as Yongyou 3, Yongyou 538, Yongyou 12, Yongyou 2640, Chunyou 84, Chunyou 927, Changyou 1, and Shenyou 1 were also released in the southern rice-growing regions. These new hybrid japonica varieties have significant advantages in grain number per panicle, yield potential, quality, plant architecture, and resistance to blast, rice bacterial blight, and rice stripe disease (Dong et al. 2016).

\section{WA -Type and Yinshui-Type Male Sterile Lines}

In the 1970s, several research institutes in China bred WAtype japonica using the three-line system (Yang and Zhu 2009). However, large-scale breeding and cultivating WAtype hybrid japonica rice was once considered impossible due to the unavailability of restorer lines. Nevertheless, some progress has been made in recent years, such as breeding japonica CMS lines Nonghu 26A and Zhen 5A, and identifying corresponding restorer lines (Zhang et al. 2003). However, the WA-type CMS lines were seldom used in agriculture because of their poor restorability, poor flowering, and pollination characteristics.

In recent years, the Chinese Rice Research Institute and LAAS have studied the Yinshui-type hybrid japonica rice, and developed three-line varieties. Importantly, heterosis and seed production technology of Yinshui-type hybrid japonica continues to be investigated.

\section{The Two-Line System for Hybrid Japonica Rice}

Studies on breeding the two-line system of hybrid japonica rice in China began in 1973 (Shi 1981). In 1985, the first japonica photo-sensitive genic male sterile (PGMS) line was generated (Luo et al. 1992). Since then, more than 20 years of research by Chinese scientists produced significant improvements in the two-line system of hybrid rice. Nuclear sterile genes of japonica lines currently available in China are mostly derived from Nongken 58S. The two-line system of hybrid japonica rice was developed rapidly in both Taihu and Yangtze valleys. Since the creation of the japonica male sterile line N5088S (used for both sterile and maintainer lines) in Hubei Province in the late 1990s, a series of similar lines, such as 7001S, has been developed and is now used for breeding. Trials and subsequent production in the Yangtze valley demonstrated that these derived cultivars have the advantages of high yield, high quality, and multi-resistance (Wang et al. 1994; Wang et al. 1995). Recently, the Northern Hybrid Japonica Research Center successfully overcame the problem of low seed setting rate of subspecies hybrids by using the two-line system. During the Chinese "Tenth Five-Year-Plan", the Anhui Academy of Agricultural Sciences used the BT-type CMS lines as the cytoplasm donor, and the japonica PGMS lines as the male maintainer to create a new SA-type sterile line. This approach not only eliminated self-fertilization of BTtype CMS lines under high-temperature conditions but also effectively prevented self-fertilization of PGMS lines under low-temperature conditions ( $\mathrm{Li}$ et al. 1997; Mou T 2016; Wang et al. 2005; Yang et al. 2008).

In northern China, GB028S was the first reported japonica PGMS line. It had the starting temperature for fertility conversion of approximately $22^{\circ} \mathrm{C}(\mathrm{Li} 1997)$. During the mid-1990s, japonica PGMS line 108S was developed to be used as sterile and maintainer lines in northern China (Wei et al. 2000).

Japonica photo-thermo-sensitive genic male sterile (PTGMS) lines were developed rapidly in the Yangtze valleys. For example, the N5088S, 31111S, and 31301S were generated in Hubei Province (Dong et al. 2016), Peiai $64 \mathrm{~S}$ in Jiangsu Province (Yuan et al. 2012), 7001S, 2304S, 8087S, and $3502 \mathrm{~S}$ in Anhui Province (Li et al. 1994; Wang et al. 2012) These PTGMS lines were used in the two-line system breeding to create a number of hybrid japonica varieties, such as 70You9, 70You4, 70Youshuangjiu, Ejingza 1, Ejingza 2, Liangyou 8828, Liangyou 122, Liangyou 276, Liangyouxinjing 1, and Liangyouxinjing 2 (Yang et al. 2009a, 2009b). The new varieties of Peiai 64S/C8420 and Peiai 64 /C418, obtained by crossing the indica PTGMS lines and japonica restorer lines with wide-compatibility genes, exhibited great potential for increasing the yield in northern China (Si et al. 2011).

\section{Progress in Genetic Studies on Traits Related to Heterosis Utilization}

Yield and Hybrid Vigor

Increasing grain yield is a long-term goal in rice breeding dictated by the need to meet the demand for global 
food security. Heterosis, i.e., higher performance for a trait in the hybrid than in both parents, offers an important strategy for rice breeding. Over the years, numerous studies have focused on the biological basis of heterosis in hybrid indica rice. It is generally believed that yield heterosis is mediated by many mechanisms, such as genetic distance (Saghai et al. 1997; Xiao et al. 1996), dominant complementary (Xiao et al. 1995), additive by additive epistatic effects (Zhuang et al. 2002), overdominance and pseudo-overdominance (Zhou et al. 2012), allele-specific expression (Lin et al. 2019), and accumulation of excellent alleles (Huang et al. 2015). Gene mapping of yield and yield-related heterosis parameters was performed in various populations, and hundreds of heterotic agronomical traits quantitative trait loci have been mapped on almost all rice chromosomes. Many QTLs contribute to heterosis, with some exhibiting strong heterotic effects on essential agronomical traits such as grain yield, flowering time, panicle grain number, seed setting rate, growth period, and photosynthetic efficiency (Chen et al. 2010; Huang et al. 2015; Huang et al. 2016; Li et al. 2016; Xin et al. 2014). Thus far, significant progress has been made in breeding hybrid japonica varieties. However, the yield of hybrid japonica has been low and was unstable in comparison with the hybrid indica. To improve hybrid vigor and combining ability (CA) between hybrid japonica parents, 81 hybrids were created, and the CA of 18 hybrids japonica parents was calculated. Associated loci residing on chromosomes 2, $5,7,9$, and 11 that recorded maximum positive values for the CA of traits were identified. It was concluded that the strategy to improve the heterosis of hybrid japonica rice involved pyramiding favorable SNP loci of $\mathrm{CA}$ and eliminating the unfavorable loci from parental genomes (Zaid et al. 2017).

\section{Flowering Time}

The yield of seed production in hybrid japonica is affected by several factors, such as physiological characteristics of parents, the technology of chemical control, cultivation methods, climate, and flowering time. For sterile lines characterized by low stigma exposure, the synchronization of flowering time with restorer lines is critical for seed production (Tong et al. 2002). The genetic factors that influence flowering time include, among others, the characteristics of the flower organ, the difference of variety type, the length and width of grain. The flowering speed of sterile lines is slower, while the flowering time occurs later than in restorer lines. Additionally, a difference in the flowering time exists between the indica and japonica varieties (Zhang et al. 2016). Typically, indica rice blooms early, reaching full flowering at about 11:00 am, while japonica rice blooms later, reaching full flowering at about 12:30 pm. Grain properties are closely related to the flowering characteristics of rice. The rounder the grain, the later it blossoms, and the longer the grain, the earlier it blossoms (Zhang et al. 2016). Due to the complexity of factors affecting the time of flowering, the research on its genetic mechanism began relatively late. Since 2010, different groups have been used to map QTLs for flower time. Using the Chuanxiang 29B/Lemont reconstituted inbred line population, three early flowering QTLs were mapped, two of them located on chromosome 10, and one on chromosome 5. The contribution rate of a major QTL reached 73.72\% (Zhang et al. 2016). Another RIL population derived from Qishanzhan/Qiuguang was used in the studies on the genetic control of flowering time. The blooming time was found to be a quantitative trait controlled by multiple genes. Six QTLs located on chromosomes $1,2,7,8,10$, and 12 were identified, with a contribution rate of $7.08 \%$ to $26.95 \%$ (Ma et al. 2011). Using the $\mathrm{F}_{2}$ population derived from WAB368-B-2-H2HB (9:31-10:00)/Liuqianxin (11:01-11:30), 4 QTLs were mapped to chromosomes $1,1,10$, and 12 , respectively, and the contribution rate of each QTL ranged from $5.8 \%$ to $11.3 \%$ (Wan et al. 2013). Recently, the CRISPR/Cas9 technique has been introduced to breed early flowering rice lines. By editing the grain length gene GS3, longgrain japonica rice was created, which had an earlier flowering time than the short-grain japonica rice. In addition, when the flowering time of the sterile line and restorer line do not coincide, hormone spraying can also provide satisfactory results. It has been demonstrated that a methyl jasmonate (MeJA) spray effectively promotes rice flowering, and indica rice is more sensitive than japonica to the treatment with MeJA, and the response of indica rice is faster than that of japonica rice. High concentration $(4 \mathrm{mmol} / \mathrm{L})$ of MeJA applied late in the afternoon, at $5: 00 \mathrm{pm}$, is the optimal treatment to promote the flowering of japonica rice (Zhang et al. 2016).

\section{Stigma Exposure Rate}

The degree of stigma exsertion is an important feature determining the seed setting rate. Increasing the seed setting rate in male sterile lines is important to achieve a high yield of hybrid seeds, and the stigma exposure rate is the main factor affecting this parameter (Dang et al. 2016). The stigma exsertion rate correlates positively with the yield of seeds; the seed setting rate in sterile lines increases by $0.74-0.92$ percentage points for every $1 \%$ increase in stigma exposure rate, equivalent to an increase of at least 47 to $68 \mathrm{~kg}$ per hectare (Yang 2016). Stigma exposure in rice is generally considered to be a quantitative trait controlled by multiple genes, with dominant inheritance, lower epistasis effect, and greater environmental influence (Li et al. 2014a, 2014b; Ma 
et al. 2018). At present, nearly 60 QTLs related to stigma exposure rate have been identified ( $\mathrm{Li}$ et al. 2014a, 2014b; Ma et al. 2018; Miyata et al. 2007; Yan et al. 2009), and are distributed throughout the 12 chromosomes of rice. Liu et al. (2015) mapped a main gene controlling the length of stigma in rice, qSTL3, to the 19.8 $\mathrm{kb}$ interval in the center of the short arm of chromosome 3 and verified the gene function. Using 227 rice germplasm as the material in the genome-wide association study, Dang et al. (2016) detected 6 QTLs regulating stigma length. Most of the stigma exsertion genes were derived from wild rice and indica subspecies, and prevalently had a low contribution rate, small additive effect, and were sensitive to the environmental conditions. In addition, the stigma exertion rate was higher in varieties with longer spikelets and longer stigma. Grain length, grain aspect ratio, stigma span, and ovary length were all positively correlated with stigma exposure rate.

\section{CMS and Fertility Restoration}

CMS, a maternally inherited inability to produce functional pollen, has been observed in more than 200 species of higher plants, and this defect is dependent on cytoplasmic genes ( $\mathrm{Hu}$ et al. 2012). Recent studies have shown male sterility caused by CMS genes are genetically bound to mitochondria. Accordingly, a protein that restores pollen sterility is encoded by nuclear genes, known as fertility restorer genes ( $R f$ genes) (Budar et al. 2003). Thus far, five rice CMS genes and eight Rf genes have been cloned (Table 2 and Table 3 ). As indicated in Table 2, all rice CMS genes are derived by the recombination process of the mitochondrial genome and are frequently coupled with the functional genes of mitochondrial respiratory chain to form a co-transcribed transcript of infertility genes (Liu et al. 2018). Among the five types of sterile lines, BT-CMS is the one most widely used in hybrid japonica rice. The BT-CMS gene orf79 was discovered in 1994 using Southern hybridization during the analysis of mitochondrial gene recombination events. The orf79 gene is located downstream of the mitochondrial Atp6 gene and encodes a protein containing 79 amino acids (Akagi et al. 2004), and its effect on fertility can be reversed by restorer lines carrying the $R f 1 a$ and $R f 1 b$ genes. $R f 1$ is the first known restorer gene for gametophytic male sterility. The BT-
CMS restorer genes $R f 1 a$ and $R f 1 b$ encode pentatricopeptide (PPR) proteins with the length of 791 and 506 amino acids, respectively. Rf1a and Rf1b contain, respectively, 18 and 11 PPR domains, and are candidate proteins for targeting mitochondria (Wang et al. 2006).

\section{Breeding Strategies \\ High Yield Breeding}

The utilization of interspecific heterosis is an effective strategy to obtain super-high yield hybrid japonica rice. In the 1960s, strong heterosis was reported in interspecies hybrids of indica-japonica, manifested mostly by tall plant height, large panicle size, large grain number, strong tillering power, strong stem strength, highly developed root system, and strong disease resistance (Yang et al. 1962). In the 1980s, breeding of indica-japonica interspecific hybrid combinations was conducted in China, resulting in the development of certain hybrid combinations, e.g., chengte232/erliuzhaizao and 3037/02428, which increased the yield by more than $20 \%$ when compared with indica rice combination Xian you 63 in the same period (Deng 2008). In recent years, a series of new high-yield indica-japonica hybrid rice combinations, such as Yongyou and Chunyou, have been bred by combining sterile lines of japonica with indica restorer lines. Ningbo seed co. LTD developed Yongyou 538, which has high and stable yield. In the provincial production test, the average yield of Yongyou 538 was 11.3 t/ha, 29.6\% higher than the control. Chunyou 84 was obtained by crossing the japonica sterile line Chunjiang $16 \mathrm{~A}$ and indica-japonica restorer line C84; it provided an average yield of $10.3 \mathrm{t} / \mathrm{ha}, 22.9 \%$ higher than the control. Nevertheless, due to significant genetic differences between the parent lines, the hybrid varieties often exhibit certain undesirable traits, such as overhigh plant (Dai et al. 1991), low seed setting rate (Zhu and Liao 1990), long growth period (Yuan 2002), and poor grain filling degree (Yuan 2002). These problems can be solved to some extent by the introduction of a widecompatibility gene or the aggregation of the specific compatibility gene $\mathrm{S}_{5}{ }^{\mathrm{i}}$ in japonica rice (Chen et al. 2011b; Mi et al. 2016; Shahid et al. 2013; Wan 2010). In northern China, in addition to using the heterosis of indica-japonica, the emphasis is placed on the introduction of the dep 1 gene in the cultivation of many hybrid

Table 2 CMS types and related genes in rice

\begin{tabular}{llll}
\hline Type & Related gene & Protein characteristics & Reference \\
\hline BT-CMS (G) & B-atp6-orf79 & Membrane protein & Akag 2004 \\
HL-CMS (G) & atp6-orfH79 & Membrane protein & Peng et al. 2010 \\
WA-CMS (S) & rpl5-WA352 & Membrane protein & Luo et al. 2013 \\
LD-CMS (G) & L-atp6-orf79 & - & Etsuko et al. 2011 \\
CW-CMS (G) & orf307 & - & Fujii and Toriyama 20 \\
\hline
\end{tabular}


Table 3 Restorers of fertility for CMS in rice

\begin{tabular}{llll}
\hline Type & Related genes & Protein characteristics & Reference \\
\hline BT-CMS (G) & Rf1a, Rf1b & Triangular pentapeptide repeat structural protein (PPR) & Wang et al. 2006 \\
HL-CMS (G) & Rf5, Rf6 & Triangular pentapeptide repeat structural protein (PPR) & Hu et al. 2012 \\
WA-CMS (S) & Rf3, Rf4 & Triangular pentapeptide repeat structural protein (PPR) & Cai et al. 2012 \\
LD-CMS (G) & Rf2 & glycine-rich protein & Etsuko et al. 2011 \\
CW-CMS (G) & Rf17 & Acyl transporter synthetase & Fujii and Toriyama 2009
\end{tabular}

japonica rice varieties with upright and semi-upright panicles, such as Liaoyou 9906 and Gangyou 1. These varieties have a higher yield, denser grain, and are less lodging than the loose panicle hybrid japonica rice (Gao et al. 2012).

\section{Quality}

Hybrid rice is produced by cross-breeding of two parents with different genotypes, the genetic mechanism of quality is very complex. It was repeatedly demonstrated that the phenotypes of the majority of quality traits in hybrid $\mathrm{F}_{1}$ tend to be of the middle parent type (Deng 2008). Therefore, when breeding high-quality japonica combinations, the selection of quality traits of both parents is essential. Choosing good quality traits of both parents can effectively prevent negative separation of hybrid rice quality (Yang 2016). For example, Jingyou 653, developed by the Liaoning Rice Research Institute, is a highquality hybrid japonica rice variety, whose parent $65 \mathrm{~A}$ and C315 are both high-quality lines. The Jingyou 653 hybrid won the first prize in the Japan-China rice tasting contest activity held in Japan in 2016.

\section{Disease Resistance}

Rice blast is a major disease in Zhejiang, Shanghai, Tianjin, Liaoning, and other regions with large planting areas of hybrid japonica rice, and has a great negative impact on rice production. Prolonged rainfall during the heading stage of rice results in the frequent occurrence of rice blast causes serious losses in rice production. The most economical and effective method to control this disease is the breeding and cultivation of blast-resistant rice varieties. To date, 36 rice blast resistance genes have been cloned. Thirty-four of these genes are dominant (Yang et al. 2019), enabling the improvement of the disease resistance of rice by gene pyramiding. The hybrid combination with strong disease resistance and broad resistance spectrum can be created by breeding and aggregating multiple japonica hybrid parents resistant to rice blast or by combining sterile lines and restorer lines with different genes. By this approach, a large number of hybrid japonica rice varieties with strong resistance and broad resistance spectrum were obtained (Chu et al. 2018). For example, the hybrid japonica rice varieties Liao73You62 carrying the Pid2 and Pid3 genes exhibited resistance to rice blast in Liaoning province for many years. Additionally, the genes of resistance to bacterial blight and rice blast were combined in the same hybrid to enhance broad resistance to various diseases (Abhilash et al. 2016; Dash et al. 2016).

\section{Combination Strategies}

The hybrid combination model plays a particularly important role in the breeding of hybrid japonica rice. During many years of breeding, the combination model of indica-japonica complementation, tiller angle complementation, yield components, morphological structure complementation, and photo/temperature complementation, has been gradually developed in China (Huang et al. 2016; Yang 2016; Yu et al. 2008). However, differences in the adopted strategies continue to be present among different regions. In the southern rice-growing regions, such as the middle and lower reaches of the Yangtze River, breeders take advantage mostly of the indica-japonica hybrid, introducing the widecompatibility genes, and selecting the hybrid varieties with big panicles, such as the Yongyou and Chunyou series. In the Huang-Huai region in central China, breeders rely mostly on the combination of photosensitive japonica male sterile lines and temperature-sensitive restorer lines to breed two-line hybrid japonica rice varieties. In the northeast rice-cultivating region, where the temperature is lower and the frost-free period is shorter, the hybrid japonica hybrid rice combinations with medium panicle grain number, more tillers, higher seed setting rate, and good synchronicity of filling are generally hybridized with multi-branched sterile lines and rice restorer lines with large panicles (Leng and Wang 2019; Yang 2016; Wang et al. 2011).

\section{Cultivation of Hybrid japonica Rice in China}

Since the approval of the first japonica hybrid rice Liyou 57 in 1980, 396 varieties have been approved until 2019 (Table 1). Although the number of approved varieties is large, only some of them can be widely cultivated due to their unstable performance or the difficulty in seed production. According to the data obtained from the $\mathrm{Na}$ tional Agro-Tech Extension and Service Center (http:// www.natesc.org.cn), by 2018, a total of 17 varieties have been cultivated to an area of more than 10,000 ha in 9 provinces in China (Table 4), among which Zhejiang 
province has the largest area, followed by Liaoning province (Fig. 4).

In the mid-1980s, the total area of japonica hybrid rice cultivation was only about 133,000 ha and accounted for just $2 \%$ of the total area of japonica rice. Even in northern China, the land used for growing hybrid japonica rice accounted for only approximately $6 \%$ of the total area of japonica rice (Xie et al. 2007). By the early 1990s, as studies on hybrid japonica rice began to decline, the planting area decreased to 80,000 ha, constituting only about $1 \%$ the of japonica rice area. However, in recent years, the area used for growing hybrid japonica rice has increased and is mainly distributed throughout Liaoning, Jiangsu, Shanghai, Zhejiang, Yunnan, and other provinces (Jiang et al. 2014; Kang 2013; Ma et al. 1998; Ni et al. 2001; Quan et al. 2000). For example, Tianlongyou 619, released by Liaoning Province Crop Variety Approval Committee in 2014 and by National Crop Variety Approval Committee in 2016, developed by a cross of a sterile line featuring more panicles and a restorer line characterized by larger panicles. Tianlongyou 619 is long-grain fragrant rice of excellent quality ("Grade I" quality according to the national standard). This variety provides grain yield of more than $9000 \mathrm{~kg} / \mathrm{ha}$, has broad adaptability, and is widely planted in Southern Jilin, Liaoning, Tianjin, Ningxia, Jiangsu, Hainan, and other provinces. The Jiangsu Academy of Agricultural Sciences developed a hybrid japonica variety 95 You161 by a cross of japonica sterile line 95122A and restorer line R161. 95You161 exhibits high quality ("Grade II") and is grown on 13,400 ha. In 2008, the Shanghai Academy of Agricultural Sciences has developed a hybrid japonica variety
Huayou 14, which was being used as a typical high-yield variety for the past 3 yrs. Huayou 14 exhibits high "Grade I" quality and is cultivated on an area of 80,000 ha. In the middle-lower Yangtze region, a number of hybrid japonica rice varieties with super-high yield and strong heterosis have been developed using indica-japonica complementation. Since 2011, a series of new indica-japonica hybrid rice varieties, including Yongyou and Chunyou, have shown high yield potential by reaching yield levels similar to those of super-high-yielding varieties (Jiang et al. 2014; Kang 2013). Zhongchunyou 84 , a subspecies hybrid rice variety with a super-high yield, was developed by a cross between the earlyblooming, sterile, dwarf japonica line Chunjiang 16A and an indica-japonica restorer line C84 with widecompatibility. From 2014 to 2015, Zhongchunyou 84 was the dominant rice variety in Zhejiang Province and was grown on an area of 46,700 ha (Dong et al. 2016). Although the planting area of hybrid japonica varieties has increased in recent years, it still accounts for less than $5 \%$ of the total japonica planting area $(\mathrm{Pu}$ et al. 2015). Due to certain key technical barriers that remain to be solved, the difference in the planting area between hybrid japonica and indica is still significant.

\section{Current Status of Genomics in Hybrid japonica}

With the development of high-throughput sequencing technology, hundreds of agronomically relevant heterotic QTLs affecting the performance of heterozygous genotypes have been mapped. Many QTLs contribute to heterosis by dominant or overdominant effects, and some exhibit strong heterotic effects on important

Table 4 List of varieties with an area of more than 100,000 ha

\begin{tabular}{|c|c|c|c|c|c|c|c|}
\hline Varieties & Female & CMS type & Male & Area(104 ha) & Type & Subspecies & Year of releasec \\
\hline Yongyou9 & Yongjing2 & BT & K6093 & 71.5 & Three-lines & Japonica & 2007 \\
\hline E jing za 1 & N5088S & BT & R187 & 61.3 & Two-lines & Japonica & 1995 \\
\hline Li you 57 & LimingA & BT & $\mathrm{C} 57$ & 59.9 & Three-lines & Japonica & 1980 \\
\hline 9 you 418 & Xu9201A & BT & C418 & 40.8 & Three-lines & Japonica & 2000 \\
\hline Yong you 6 & Yongjing2A & BT & K4806 & 28.9 & Three-lines & Japonica & 2005 \\
\hline Han you xiang qing & HanfengA & BT & Xiangqing & 25.7 & Three-lines & Japonica & 1989 \\
\hline Yong you 12 & Yongjing2A & BT & F5032 & 25.7 & Three-lines & Japonica & 2010 \\
\hline Yong you 1 & Ning67A & BT & K1722 & 20.7 & Three-lines & Japonica & 2000 \\
\hline Xiu you 57 & XiulingA & BT & $\mathrm{C} 57$ & 20.4 & Three-lines & Japonica & 1984 \\
\hline Wan dao 26 & 70015 & BT & Xiushui04 & 19.3 & Two-lines & Japonica & 1994 \\
\hline Wan dao 34 & $80-4 A$ & BT & HP121 & 13.9 & Three-lines & Japonica & 1996 \\
\hline Yong you 538 & Yongjing3A & BT & F7538 & 13.7 & Three-lines & Indica-japonica & 2013 \\
\hline III you98 & $\mathrm{MH} 2003 \mathrm{~A}$ & BT & R18 & 13.4 & Three-lines & Japonica & 2002 \\
\hline E jing za 3 & N5088S & BT & Minhui128 & 13.3 & Two-lines & Japonica & 2004 \\
\hline Yong you 17 & Yongjing4A & BT & Yonghui12 & 11.1 & Three-lines & Indica-japonica & 2012 \\
\hline Chun you 59 & Chunjiang16A & BT & $\mathrm{CH} 59$ & 10.7 & Three-lines & Japonica & 2009 \\
\hline
\end{tabular}




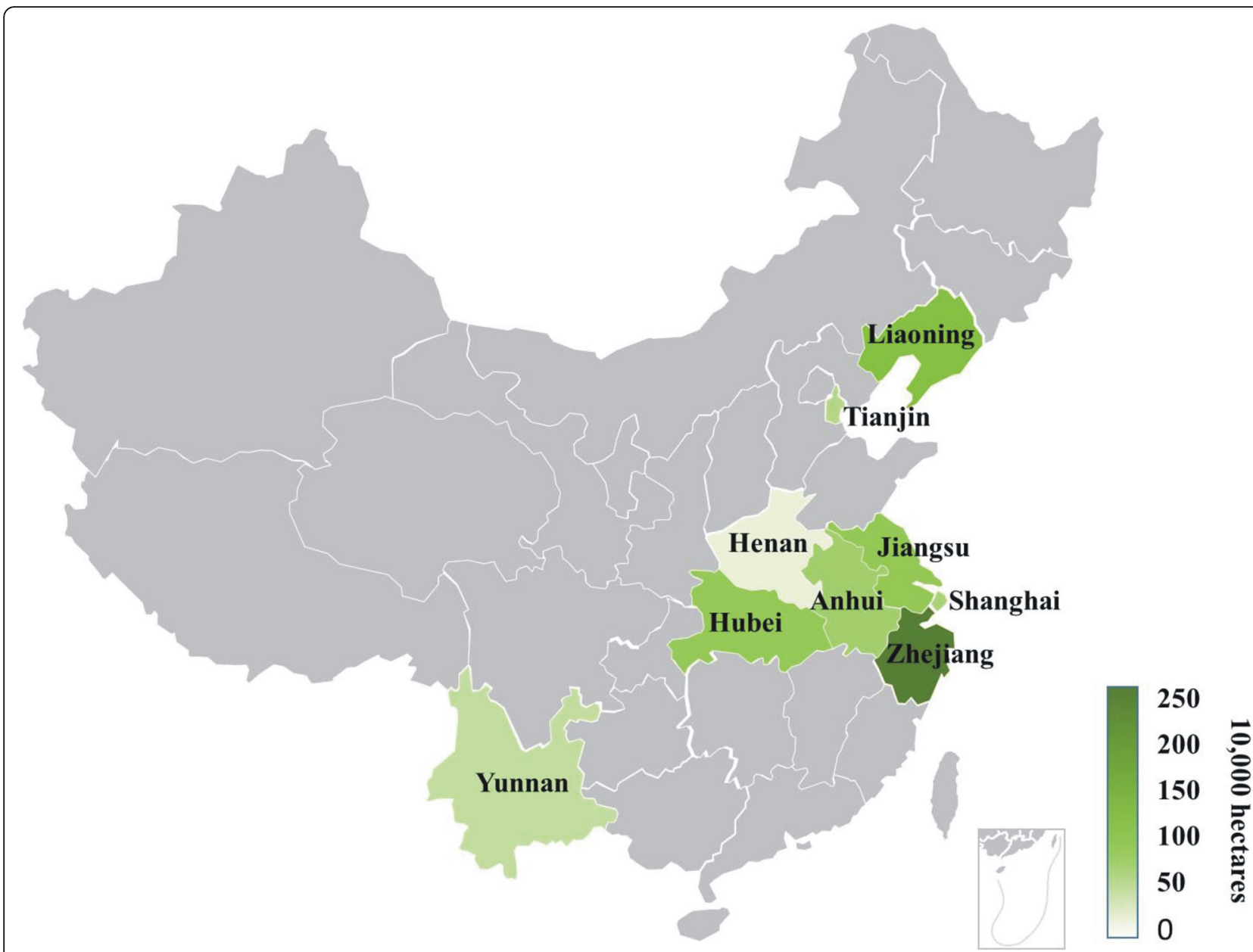

Fig. 4 Production map of hybrid japonica rice of China. The provinces marked green are those with a cumulative promotion area of more than 10,000 ha from 1981 to 2018. The deeper the green degree, the larger the cumulative promotion area

agronomical characteristics such as grain yield and flowering time (Huang 2015; Huang 2016; Li et al. 2016; Lin et al. 2019). At the same time, the molecular mechanism of heterosis was gradually elucidated by transcriptome sequencing and genome resequencing technique. In 2015, DNA sequencing of 1495 elite hybrid rice varieties and their inbred parental lines was performed. Comprehensive analyses of heterozygous genotypes revealed that heterosis results mostly from the accumulation of numerous superior alleles with positive dominant effects (Huang et al. 2015). Huang et al. (2016) generated the sequences and recorded the phenotypes of $10,074 \mathrm{~F}_{2}$ lines from 17 representative hybrid rice crosses. They documented that a small number of genomic loci from female parents are responsible for a large part of the yield advantage that hybrids have over their male parents. For some of those loci, they found support for partial dominance of heterozygous locus for yield-related traits when all grain-yield traits were considered together. In the process of hybrid rice breeding, breeders tend to introduce different introgressed exogenous genomes unconsciously to shaped heterotic loci in the hybrid rice. Lin et al. (2020) generated two populations of rice $F_{1}$ hybrids using commercial hybrid parents and genotyped the parents by a $50 \mathrm{k}$ SNP chip and genome resequencing, the results from the analysis revealed that the male and female parents have different levels of genome introgressions from other rice subpopulations, including indica, aus, and japonica, therefore shaping heterotic loci in the hybrids. Among the introgressed exogenous genome, heterotic loci, including Ghd8/DTH8, Gn1a, and IPA1 existed in wild rice, but were significantly divergently selected among the rice subpopulations, suggesting these loci were subject to environmental adaptation. During modern rice hybrid breeding, heterotic loci were further selected by removing loci with negative effect and fixing loci with positive effect and pyramid breeding. These findings may facilitate future breeding of improved varieties of hybrid rice (Lin et al. 2020). However, these experimental studies 
utilized mostly indica hybrid rice or their parents, while the understanding of the genomics of hybrid japonica rice is still lagging behind.

\section{Problems and Prospects}

Under the leadership of Academician Longping Yuan and the joint efforts of several rice-breeding institutes in China, remarkable progress was made in the development of hybrid japonica rice. However, some problems restricting further improvements remain in place, necessitating collaborative research. First, the yield advantage of hybrid japonica rice is not high enough in comparison with traditional japonica rice. On the one hand, due to the limited knowledge of the genetic background of the parents of japonica hybrid rice, the genetic distance between the parents of japonica hybrid rice is not sufficiently large, leading to a weak yield heterosis. On the other hand, the introduction of the indica genome into hybrid japonica rice was typically considered as a strategy to enhance the heterosis. However, under the influence of the indica genetic background, hybrid japonica rice often exhibit premature senescence and differentiation between strong and weak (filled and partiallyfilled) grains. In addition, with low temperature present during the later growth stages, the weak grains are not sufficiently filled and do not mature fully, reducing the yield potential. Therefore, to avoid the negative effects of the introduction of indica rice genetic background, further research should focus on the mechanism of the formation of filled and partially-filled grains and the inheritance of cold tolerance. Second, despite the important breakthroughs and developments in mechanized seed production accomplished by the NJETC and the Shanghai Academy of Agricultural Sciences, the low yield of hybrid japonica seeds is the key limiting factor in the applications of hybrid japonica. Due to the fact that the restore genes were derived mostly from indica rice, the restorer lines with some indica background have early blooming time. However, most japonica sterile lines exhibit late blooming time and low percent of stigma exposure. Moreover, with the development of directly seeded rice, the need for an increased amount of seeds created a new challenge for the production of hybrid japonica rice seeds. Therefore, it is necessary to accelerate germplasm screening and gene mining, and utilize these data to achieve early blooming time, high stigma exposure rate, large stigma size, and strong stigma vigor.

It should also be noted that the growth and development of hybrid japonica rice are different from conventional japonica rice. Cultivation techniques should be developed according to the characteristics of hybrid japonica rice. The hybrid japonica rice possesses larger panicles and more grains, and it was usually faced with large sink but small source. In terms of cultivation, an adequate population structure should be established in the early and middle stages of growth to ensure sufficient and effective number of panicles and spikelets, and to avoid the overgrowth of plants. Attention should be given to ensuring grain filling and preventing premature senescence by postponing panicle fertilizer. To fully utilize the yield potential of hybrid japonica rice, early sowing and transplanting should be implemented to ensure that the heading and grain-filling occur at the most opportune period.

Finally, the cooperation between scientific research institutions and seed enterprises should be enhanced to jointly promote the commercial operation and industrial development of hybrid japonica rice, accelerate the expansion and application of new hybrid japonica rice varieties, and seize the advantages of hybrid japonica rice such as reduced use of fertilizer and water, strong resistance, and high yield. These steps will improve the utilization of middle- and low-yield fields and ensure food security.

\section{Conclusions}

With the increasing demand for high-quality japonica rice, the prospects for the development of hybrid japonica rice are increasingly better, particularly in China. Although the number and the spread area of hybrid japonica rice varieties lag behind hybrid indica, the research on hybrid japonica rice progressed remarkably over the past 70 years. Several male sterile lines (e.g., Dian-type, BT-type, WA -type, and Yinshui-type) with their corresponding restorer and maintainer lines have been used in the three-line system of growing japonica hybrid rice. The development of photo-thermo-sensitive genic male sterile lines for the two-line system also promises great potential for improving grain yield in hybrid japonica rice. Meanwhile, remarkable progress has been made in research on molecular mechanisms for heterosis, stigma exposure rate, flowering time, and male sterility in hybrid japonica. In future, exploiting and pyramiding the superiority genes with yield-related genes by MAS will have an important role in increasing grain yield of hybrid japonica. Given the present limitations, we have proposed four effective strategies to develop hybrid japonica: (1) increasing parental genetic distance and introducing the wide-compatibility genes; (2) accelerating germplasm screening, gene mining, and utilizing the data to achieve early blooming time, high stigma exposure rate, large stigma size, and strong stigma vigor; (3) using new cultivation techniques specific for hybrid japonica; and (4) enhancing cooperation with extension departments and cooperatives. 


\section{Acknowledgements}

Not applicable.

\section{Authors' Contributions}

All authors listed have made a substantial, direct, and intellectual contribution to the work and approved it for publication.

\section{Funding}

This work was supported by the National Key R\&D Program of China (2017YFD0300700), Liao Ning Revitalization Talents Program (XLYC1802119) and Liaoning Key agricultural program (2019JH1/10200001-2).

\section{Availability of Data and Materials}

Not applicable.

\section{Ethics Approval and Consent to Participate}

Not applicable.

\section{Consent for Publication}

The manuscript has been approved by all authors.

\section{Competing Interests}

The authors declare that the research was conducted in the absence of any commercial or financial relationships that could be construed as a potential conflict of interest.

\section{Author details}

${ }^{1}$ Institute of Rice Research, Liaoning Academy of Agricultural Sciences, Shenyang 110000, China. ${ }^{2}$ Heilongjiang Academy of Agricultural Sciences, Haerbin 1550086, China. ${ }^{3}$ Liaoning Academy of Agricultural Sciences, Shenyang 110161, China.

Received: 5 December 2019 Accepted: 28 May 2020

Published online: 08 June 2020

\section{References}

Abhilash K, Balachiranjeevi C, Bhaskar N et al (2016) Development of genepyramid lines of the elite restorer line, RPHR-1005 possessing durable bacterial blight and blast resistance. Front Plant Sci 7:1-15

Akagi H, Nakamura A, Yokozeki-Misono Y et al (2004) Positional cloning of the rice Rf-1 gene, a restorer of $\mathrm{BT}$ type cytoplasmic male sterility that encodes a mitochondria-targeting PPR protein. Theor Appl Genet 108(8):1449-1457

Budar F, Touzet P, De Paepe R (2003) The nucleo-mitochondrial conflict in cytoplasmic male sterilities revisited. Genetica 117(1):3-16

Cai J, Liao Q, Dai Z et al (2012) Allelic differentiation and effects of the Rf3 and Rf4 genes on fertility restoration in rice with wild abortive cytoplasmic male sterility. Biol Plant 57. https://doi.org/10.1007/s10535-012-0294-9

Cao L, Cheng C, Zhou J et al (2018) Development and prospect of hybrid japonica rice industry in Shanghai. China Seed Industry 9:19-22

Chen A, Hua Z, Li Z (2011a) Inheritance analysis and detection of QTLs for exserted stigma rate in rice. J Shenyang Agric Univ 6:142-146

Chen L, Zhao Z, Liu X et al (2011b) Marker-assisted breeding of a photoperiodsensitive male sterile japonica rice with high cross-compatibility with indica rice. Mol Breed 27:247-258

Chen S, Shen X, Cao Let al (2010) QTL mapping for heterosis of yield traits in rice. Sci Agric Sin 43(24): 4983-4990

Chu H, Cheng C, Niu F et al (2018) Distribution of 8 rice blast-resistant genes in parents of three-line hybrid keng rice. Acta Agric Shanghai 34(1):8-13

Dai KG, Kang CL, Fu J (1991) Preliminary study on stalk height of indica-japonica interspecies combinations. Hybrid rice 6:31-34

Dang X, Liu E, Liang Y et al (2016) QTL detection and elite alleles mining for stigma traits in Oryza sativa by association mapping. Front Plant Sci 7:1188

Dash A, Rao R, Rao G et al (2016) Phenotypic and marker-assisted genetic enhancement of parental lines of rajalaxmi, an elite Rice hybrid. Front Plant Sci 7:1005

Deng H (2008) Japonica hybrid rice in China. China Agric Press, Beijing

Dong L, Li Z, Zhang P (2016) Progress and prospect of hybrid japonica rice breeding in China. China Rice 22:1-5

Etsuko I, Natsuko I, Sota F, Tomohiko K, Kinya T (2011) The fertility restorer gene, rf2, for lead rice-type cytoplasmic male sterility of rice encodes a mitochondrial glycine-rich protein. Plant J 65(3):359-367
Fang F (2005) Current situation of japonica rice production in China and the world. World Agric 12:22-23

Fujii S, Toriyama K (2009) Suppressed expression of RETROGRADE-REGULATED MALE STERILITY restores pollen fertility in cytoplasmic male sterile rice plants. Proc Natl Acad Sci U S A 106(23):9513-9518

Gao H, Lin H, Sun J et al (2012) Molecular basis of utilization of indica-japonica hybridization to japonica super-rice breeding in North China. J Shenyang Agric Univ 43(4):394-400

Hu J, Wang K, Huang W et al (2012) The rice pentatricopeptide repeat protein RF5 restores fertility in Hong-Lian cytoplasmic male-sterile lines via a complex with the glycine-rich protein GRP162. Plant Cell 24(1):109-122

Hu P (2010) Development and technological innovation of hybrid rice. Industry J of Agric Sci Tech 12:17-23

Hua Z, Yuan X, Sui G (2006) Genetic improvement and physiological basis of hybrid japonica rice in North China. Liaoning Sci Tech Press, Shenyang

Hua Z, Zhang Z, Wang Y et al (2002) Progress in breeding super hybrid japonica rice in North China. China Rice 2:30-31

Huang D (2004) New progress has been made in the breeding of Yunnan japonica hybrid rice. J Yunnan Agric Univ 19:665

Huang X, Yang S, Gong J et al (2015) Genomic analysis of hybrid rice varieties reveals numerous superior alleles that contribute to heterosis. Nat Commun 6:6258

Huang X, Yang S, Gong J et al (2016) Genomic architecture of heterosis for yield traits in rice. Nature 537:629-633

Jiang Y, Zhang H, Zhao K et al (2014) Difference in yield and its components characteristics of different type rice cultivars in the lower reaches of the Yangtze River. Chin J Rice Sci 28:621-630

Kang Z (2013) The development report of "Yongyou" series hybrid rice is a great innovation in the history of rice development in China. Ningbo Communication 1:47-48

Leng C, Wang Y (2019) Development strategy of japonica hybrid rice study in Heilongjiang province. Heilongjiang Agric Sci 10:132-136

Li C, Xu K, Wang S et al (1994) Study on the fertility of japonica PGMR 7001S and its utilization. J Anhui Agric Sci 22(1):11-15

Li D, Huang Z, Song S et al (2016) Integrated analysis of phenome, genome, and transcriptome of hybrid rice uncovered multiple heterosis-related loci for yield increase. Proc Natl Acad Sci U S A 113(41):6026-6035

Li J, Li ZY, Guo W (1997) Breeding of japonica PGMS line GB028S in rice. Hybrid Rice 4:4-6

Li P, Feng F, Zhang Q et al (2014a) Genetic mapping and validation of quantitative trait loci for stigma exsertion rate in rice. Mol Breeding 34(4): 2131-2138

Li P, Su G, Feng Fet al (2014b). Mapping of minor quantitative trait loci (QTLs) conferring fertility restoration of wild abortive cytoplasmic male sterility and QTLs conferring stigma exsertion in rice. Plant Breed 133: 722-727

Li T (1987) Genetic study on stigma extrusion of rice. Acta Agron Sin 13:314-321

Li Z, Wu J (1991) The present situation and prospect of three-line hybrid japonica rice breeding in China. Hybrid Rice 15:13-16

Li ZY (1977) Utilization of heterosis in rice. Agricultural Press, Beijing, pp 29-44

Li ZY (2000) Dian-type hybrid rice breeding. Yunnan Science and Technology press, Kunming, pp 1-15

Lin S, Feng X, Cong H et al (2019) Patterns of genome-wide allele-specific expression in hybrid rice and the implications on the genetic basis of heterosis. Proc Natl Acad Sci U S A 116(12):5653-5658

Lin Z, Qin P, Zhang X et al (2020) Divergent selection and genetic introgression shape the genome landscape of heterosis in hybrid rice. Proc Natl Acad Sci U S A 117(9):4623-4631

Ling Z (1989) Study on stigma exsertion after anthesis in rice II.The inheritance of stigma exsertion in some rice crosses. J China Agric Univ 15:23-27

Liu Q, Qin J, Li T et al (2015) Fine mapping and candidate gene analysis of aSTL3, a stigma length-conditioning locus in rice (Oryza sativa L). PLoS One 10: 0127938

Liu S, Chen Q, Hong G et al (2018) Research progress of cytoplasmic male sterility and fertility restoration in rice (Oryza sativa). Plant Physiology Journal 54(1):1-9

Luo D, Xu H, Liu Z et al (2013) A detrimental mitochondrial-nuclear interaction causes cytoplasmic male sterility in rice. Nat Genet 45(5):573-577

Luo X, Qiu Z, Li R (1992) Pei-Ai64S a dual-purpose sterile line whose sterility is induced by low critical temperature. Hybrid Rice 7:27-29

Ma X, Zheng Z, Lin F et al (2018) Genetic analysis and gene mapping of a low stigma exposed mutant gene by high-throughput sequencing. PLoS One 13: 0186942 
Ma Z, Liu X, Sun L et al (1998) Effect of several main problems on developing japonica hybrid rice in Tianjin. J Tianjin Agric Sci 4:55-56

Ma Z, Zhan Z, Cheng X et al (2011) Flowering time in filial generations of cross between indica and japonica rice and its response to external environment. Hybrid rice 26(5):70-76

Mi J, Li G, Huang J et al (2016) Stacking S5- $n$ and $f 5-n$ to overcome sterility in indica-japonica hybrid rice. Theor Appl Genet 129:563-575

Miyata M, Yamamoto T, Komori T, Nitta N (2007) Marker-assisted selection and evaluation of the QTL for stigma exsertion under japonica rice genetic background. Theor Appl Genet 114:539-548

Mou T (2016) The research progress and prospects of two-line hybrid rice in China. Chin Sci Bull 61:3761-3769

Ni L, Yuan Q, Cao L, Wu Y (2001) Genetic analysis of rice quality characters of hybrid japonica varieties in shanghai area. Acta Agric Shanghai 17:22-26

Peng X, Wang K, Hu C et al (2010) The mitochondrial gene orfH79 plays a critical role in impairing both male gametophyte development and root growth in CMS-Honglian rice. BMC Plant Biol 10:125

Pu H, Zhou Z, Xu D (2015) Development history and problems of three-line hybrid japonica rice. J Jiangsu Agric Sci 43:74-77

Quan L, Shi B, Gao W, Yuan Q (2000) An approach to japonica hybrid rice quality breeding in shanghai region. Acta Agric Shanghai 16:21-23

Saghai M, Yang G, Zhang Q et al (1997) Correlation between molecular marker distance and hybrid performance in US southern long grain rice. Crop Sci 37 $145-150$

Sampath S, Mohauty H (1954) Cytology of semi-sterile rice hybrids. Curr Sci 23: 182-183

Shahid M, Chen F, Li H et al (2013) Double-neutral genes, Sa-n and Sb-n, for pollen fertility in rice to overcome indica $\times$ japonica hybrid sterility. Crop Sc 53:164-176

Shen S, Yan J, Zhang N (1994) Genetic analysis for some floral characters in hybridization between indica and japonica rice. Acta Agric Zhejianggensis 7: 222-225

Shi M (1981) Preliminary report on breeding and application of late japonica natural dual-use lines. J Hubei Agric Sci 7:1-3

Shiniyo C, O' mura T (1966) Cytoplasmic male sterility in cultivated rice, Orayza sativa $L$. I. Fertility of $F_{1}, F_{2}$, and offsprings obtained from their mutual reciprocal backcrosses; and segregation of completely male sterile plants. Jpn. J. Breed 16(suppl.) 1: 179-180

Shiniyo C (1969) Cytoplasmic-genetic male sterility in cultivated rice, Orayza sativa L. J Genet 44(3):149-156

Shiniyo C (1972) Distribution of male sterility introducing cytoplasm and fertility restoring genes in rice. I commercial lowland rice cultivated in Japan Jpn JGenet 47: 237-243

Si H, Liu W, Fu Y et al (2011) Current situation and suggestions for development of two-line hybrid rice in China. China J Rice Sci 25:544-552

Tang L, Xu Z, Chen W et al (2017) Advances and prospects of super rice breeding in China. J Integr Agric 16(5):984-991

Tong H (2002) Factors affecting seed yield and quality of hybrid rice. Sci Tech Seeds 1:33-34

Wan G, Feng Y, Zhang F et al (2013) Mapping QTLs for flowering time in rice. J of Nuclear Agricult Sci 27(5):0562-0567

Wan J (2010) Utilization of strong heterosis between indica andjaponca varieties in Rice. Proceedings of the first hybrid Rice congress in China 3-6

Wang C, Feng Y, Qi H (1994) Identification and evaluation of japonica photoperiod sensitive genic male sterile line N5088S. China Seed Industry 4: 20-21

Wang C, Qi H, Feng Y et al (1995) Selection and application of japonica photoperiod sensitive genic male sterile line N5088S. Hubei Agric Sci 6:5-12

Wang C, Wu T, Zhang Y (2011) Analysis of relativity between main quality traits, combining ability and heterosis of hybrid rice in North China. Southwest China J Agric Sci:17-22

Wang S, Du S, Wu S et al (2005) Development and study of japonica male sterile lines integrating cytoplasmic male sterility and photosensitive genic gale gterility. Sci Agric Sin 38(7):1289-1294

Wang S, Du S, Wu S et al (2012) Breeding and characteristics of photoperiod sensitive genic male sterile line wan 2304 S in japonica rice. J Anhui Agric Sci 40:3959-3960

Wang Y, Hua Z, Zhang Z et al (2008) Breeding and application of japonica male sterile lines with high stigma exsertion rate in Rice. Hybrid Rice 23:4-8

Wang Z, Zou Y, Li X et al (2006) Cytoplasmic male sterility of rice with Boro II cytoplasm is caused by a cytotoxic peptide and is restored by two related
PPR motif genes via distinct modes of mRNA silencing. Plant Cell 18(3):676687

Wei Y, Yang Z, Zhang Z et al (2000) Breeding and application of an early japonica PTGMS line 108S. Hybrid Rice 15(supplement): 1-2

Xiao J, Li J, Yuan L et al (1995) Dominance is the major genetic basis in rice as revealed by QTL analysis using molecular markers. Genetics 140(2):745-754

Xiao J, Li J, Yuan L et al (1996) Genetic diversity and its relationship to hybrid performance and heterosis in rice as revealed by PCR-based markers.Theor. Appl Genet 92(6):637-643

Xie F, Peng S (2016) History and prospects of hybrid rice development outside of China. Chinese Sci Bulletin 61:3858-3868

Xie H, Jing Y, Zeng Q et al (2007) Necessity and trend of hybrid japonica rice development in China. North Rice 3:10-13

Xin Y, Yuan L (2014) Heterosis loci and QTL of super hybrid rice liangyoupeijiu yield by using molecular marker. Sci Agric Sin 47(14):2699-2271

Yan W, Li Y, Agrama H et al (2009) Association mapping of stigma and spikelet characteristics in rice (Oryza sativa L). Mol Breeding 24:277-292

Yang D, Wang M, Han L et al (2019) Progress of cloning and breeding application of blast resistance genes in rice and avirulence genes in blast fungi. Chinese Bulletin of Botany 54(2):265-276

Yang J, Wang J, Cao Q (2009a) Development and application of a functional marker for wide compatibility gene S5-n of rice. Acta Agron Sin 35:20002007

Yang J, Zhu X (2009) Research and breeding progress of WA type hybrid rice. China Rice 6:1-3

Yang L, Wu W, Bai Y (2008) 80 you 1, a new medium japonica hybrid rice combination with good grain quality. Hybrid Rice 23:80-81

Yang S, Cheng B, Shen W et al (2009b) Progress of application and breeding on two-line hybrid rice in China. Hybrid Rice 24:5-9

Yang S, Shen K, Gu W (1962) Studies on rice cross-breeding between indica and japonica. (II). Acta Agron Sin 1(2):97-102

Yang Z (1994) Progresses in the breeding of japonica hybrid rice. Hybrid Rice S1: $46-49$

Yang Z (1998) Retrospects and prospects on the development of japonca hybrid rice in the north of China. Acta Agron Sin 24:840-846

Yang Z (1999) Breeding of hybrid japonica rice in North China. China Agricultural Science and Technology Press, Beijing, pp 21-27

Yang Z (2005) Development and technical strategy of japonica hybrid rice in China. Hybrid Rice 20:5-6

Yang Z (2016) Development and prospect of hybrid japonica rice in China (in Chinese). China Sci Bull 61:3770-3777

Yu C, Jiang L, Xiao Y, Zhai H, Wan J (2008) Combining ability of yield-component traits for indica chromosome substituted segments in japonica hybrids. Acta Agron Sin 34:1308-1316

Yuan D, Tan Y, Yu D et al (2012) Improving the blast resistance of Pei-ai-64S by marker-assisted selection. Molecular Plant Breeding 3:278-284

Yuan L (1987) Breeding strategy of hybrid rice. Hybrid Rice 1:1-3

Yuan L (2000) Super-high yield breeding of hybrid rice. Hybrid Rice 15:31-33

Yuan L (2002) Hybrid rice science. China Agric Press, Beijing

Zaid I, Tang W, Liu E et al (2017) Genome-wide single-nucleotide polymorphisms in CMS and restorer lines discovered by genotyping using sequencing and association with marker-combining ability for 12 yield-related traits in Oryza sativa L. subsp. Japonica. Front. Plant Sci 8:143

Zhang A, Xu L, Jin Y, Ning H (2003) Study on three-line system and genetic mechanism of WA type japonica. J Anhui Agric Sci 31(17-18):21

Zhang M, Dai D, Li X, Zhang H, Ma L (2016) Advances on the study of flowering time trait in hybrid rice. J Nuclear Agric Sci 30:0267-0274

Zhang P, Xu W, Yuan P et al (1999) Studies on the breeding way of medium japonica hybrid rice combination between sub-subspecies with the superhigh yield. J Anhui Agric Sci 27(305-306):308

Zhou G, Chen Y, Yao W et al (2012) Genetic composition of yield heterosis in an elite rice hybrid. Proc Natl Acad Sci 109(39):15847-15852

Zhu Y, Liao F (1990) Advances in research on interspecific heterosis of two-line in hybrid rice. Hybrid rice 3:32-34

Zhuang J, Fan Y, Rao Z et al (2002) Analysis on additive effects and additive by additive epistatic effects of QTLs for yield waits in a recombinant inbred line population of rice. Theor Appl Genet 105:1137-1145

\section{Publisher's Note}

Springer Nature remains neutral with regard to jurisdictional claims in published maps and institutional affiliations. 